\title{
Astrometric meaning and interpretation of high-precision time delay integration CCD data ${ }^{\star}$
}

\author{
U. Bastian ${ }^{1}$ and M. Biermann ${ }^{2}$ \\ 1 Astronomisches Rechen-Institut, Mönchhofstr. 12-14, 69120 Heidelberg, Germany \\ e-mail: bastian@ari.uni-heidelberg.de \\ 2 Landessternwarte Heidelberg, Königstuhl 12, 69117 Heidelberg, Germany
}

Received 16 November 2004 / Accepted 16 February 2005

\begin{abstract}
We investigate the astrometric content of CCD charge images of stars collected in time delay integration (TDI) mode with a scanning (rotating) telescope. We focus on the ESA astrometric space mission Gaia, but the results are valid for other scanning telescopes too. The physical attitude of the telescope is shown to be unobservable. Instead, an effective astrometric attitude is observed which represents an average over the TDI exposure time. The effective astrometric attitudes "seen" by different instruments (in case of Gaia: Astro, Spectro, Astro with gates) differ in a non-trivial way. If e.g. the high-precision Astro attitude would be used for the astrometric exploitation of the Spectro data, the Spectro CCDs would be "seen" to float around on the focal plane by several milli-arcseconds. In addition we find that the TDI mode produces an attitude jitter with the period of TDI clocking. We prove that this is negligibly small in the case of Gaia. We point out that the effective instant of observation is not the instant of charge read-out from the CCDs, but about half an exposure time (i.e. up to a few seconds) earlier. This is particularly important for the astrometry of solar-system objects and for the photometry of rapidly varying objects. It is also relevant for all other objects because of the time dependence of aberration. It is not clear whether the differences between the astrometric attitudes of different instruments require separate attitude reconstructions, but an approximate transformation from Astro to Spectro probably will be sufficient.
\end{abstract}

Key words. astrometry - methods: data analysis - space vehicules: instruments

\section{Introduction}

\subsection{Scanning CCDs in TDI mode}

CCD detectors allow the recording of sharp long-exposure images from rotating platforms. This is achieved by letting the optical image of the observed object(s) move across the detector chip at constant speed (determined by the rotation rate of the platform and the focal length of the optics) and transporting the gradually accumulating photo-electric charges at the same speed by appropriate clocking of the "parallel" charge transport mechanism of the CCD.

This method is called Time Delay Integration (TDI) mode, or drift-scan mode. The final image of the observed object(s) is recorded when the image reaches the edge of the $\mathrm{CCD}$ where the serial register (read-out register) is located. The effective exposure time is the time it takes the optical image to cross the entire light-sensitive area of the CCD.

The TDI mode is used in scanning spacecraft (astronomical or Earth-observing ones), as well as in ground-based astronomical survey telescopes. In the latter case it is the daily rotation of the Earth that creates the scanning motion.

* Appendices $\mathrm{A}$ and $\mathrm{B}$ are only available in electronic form at http://www . edpsciences.org
A characteristic consequence of the TDI mode is that the CCDs do not produce separate "frames" having as many data pixels as there are physical pixels on the chip. Instead, a "semi-infinite", continuous strip of pixels is delivered to the user. The width of this strip (in units of pixels) is equal to the number of pixel columns that the CCD has. The length of the strip is limited only by the contiguous stretch of time during which the TDI mode is operated.

The CCDs on board Gaia will be operated in TDI mode.

\subsection{Historical background}

Transit instruments are the major observational devices for global (i.e. large-angle) high-precision measurements. This started with mural quadrants, continued through visual filar micrometers on meridian circles, photoelectric slit micrometers on meridian circles and astrolabes, and culminated in the slit systems of the Hipparcos and Tycho instruments in space.

In all these sky-scanning systems the location of the instantaneous optical image of a star was continuously compared to some fiducial line(s) in the instrument. So, the geometric interpretation of a measurement was relatively simple. The transit 
time $t_{\mathrm{tr}}$, being the primary observable, is determined by three quantities:

- the along-scan angular coordinate $\psi_{*}$ of the star on the sky;

- the along-scan attitude $\psi_{\mathrm{i}}$ of the instrument;

- the along-scan angular location $\eta_{\text {tr }}$ of the fiducial line within the instrument

such that $\psi_{*}\left(t_{\mathrm{tr}}\right)-\psi_{\mathrm{i}}\left(t_{\mathrm{tr}}\right)-\eta_{\mathrm{tr}}=0$. All three quantities in this equation will be precisely defined below; the instrumental calibration quantity $\eta_{\text {tr }}$ does not carry a time argument here, since it must be assumed to be essentially constant over some prolonged interval of time.

In a scanning TDI instrument the optical star images move across a CCD chip, exit the trailing side of the chip and are recorded in the serial register of the chip at that time. Thus, one is tempted to identify the trailing edge of the CCD with the fiducial line of the other transit instruments, and the instant of read-out into the serial register with the transit time $t_{\mathrm{tr}}$. Indeed, the astrometric observation equations for Gaia are conventionally formulated in this way.

However, in all aforementioned instruments the duration of an elementary integration of the moving star image is shorter than the duration of its actual transit over the fiducial line. This means that in those cases, truly "instantaneous" attitude and star positions are recorded at the transit time $t_{\mathrm{tr}}$.

In a scanning CCD instrument this is no longer true. The moving star image covers a few (typically 3 to 8) CDD pixels along scan, in order to satisfy the sampling theorem (thus not to lose essential spatial information). This means that it will be registered within a few TDI clock intervals, while exiting the CCD chip. Before that, it will typically have travelled thousands of pixels across the CCD, gradually accumulating photoelectric charge on the chip. This means that the registered image will represent the average of the star's position and the instrument's scanning motion over a much longer interval of time. In other words, it is no longer representative of the "instantaneous" attitude and star position at the transit time $t_{\mathrm{tr}}$.

\subsection{Goals of this paper}

In the following we show that the convential formulation of the astrometric observation equations can be kept if the quantities contained in them are properly defined and interpreted. We discuss the consequences of this approach and identify a number of important implications.

Section 2 presents some of the basic concepts and notations needed for the present work.

In Sect. 3 we introduce the central question on the precise astrometric meaning of TDI data. The basic equations containing the answer to this question are derived.

In Sects. 4-7 we discuss the major consequences for the astrometric attitude modelling, astrometric source modelling and photometric source modelling.

Section 8 briefly discusses the results and summarises the practical consequences for Gaia.

In Appendix A we give a more complete model of the TDI data by removing some of the simplifications made for clarity and simplicity in Sects. 4-7. Some of the results give an a posteriori justification for the earlier simplifications.

Appendix B contains mathematical details for a subject touched on in Sect. 4.

\subsection{Application to Gaia}

The results of this paper will be applied to the specific case of Gaia, ESA's future astrometric and photometric survey mission (ESA 2005). The present baseline technical design for this mission comprises two instruments called "Astro" and "Spectro". The Astro instrument performs high-precision astrometric measurements, plus broad-band photometry, the latter in a specific focal-plane section called Broad-Band Photometer (BBP). The Spectro instrument consists of a Medium-Band Photometer (MBP) and a Radial-Velocity Spectrograph (RVS). All observations are carried out in TDI mode. Due to different focal lengths of the telescopes $(46.70 \mathrm{~m}$ and $2.3 \mathrm{~m}$, respectively) and different along-scan sizes of the CCDs (4500 and 800 pixels of $10 \mu \mathrm{m}$, respectively) the exposure times per CCD are different: $3.3 \mathrm{~s}$ for Astro and $12 \mathrm{~s}$ for Spectro. This is the relevant difference between the instruments for the subject of the present paper.

As the images of bright stars (from about 12th magnitude) would saturate with a $3.3 \mathrm{~s}$ exposure time on the Astro instrument, special electrodes called "gates" on the CCDs will be used to shorten their exposure. There are 12 gates in total, providing essentially a power-of-two series of different exposure times, from very short (e.g. $0.006 \mathrm{~s}$ for gate No. 3 ) to intermediate (e.g. $0.38 \mathrm{~s}$ for No. 9) and to almost the full CCD (1.5 s for gate No.11). The results of the present paper will indicate that precision astrometry using gates will be highly problematic.

For a more detailed description of the technology, mission planning and science goals of Gaia see ESA (2005).

\section{Basic concepts and notations}

As a prelude to the definition of spacecraft attitude we here briefly recall a number of basic concepts.

\subsection{Coordinate systems}

\subsubsection{Equatorial coordinates; the International Celestial Reference System (ICRS)}

Star positions, or more generally, directions in the astronomical sense are given in some celestial coordinate system. Conventionally this is the International Celestial Reference System (ICRS). The spherical longitude and latitude coordinates in this system are called right ascension $(\alpha)$ and declination $(\delta)$. The fundamental plane $(\delta=0)$ and the zero point $(\alpha=0)$ of the ICRS closely match the earth's equatorial plane and the direction to the dynamical spring equinox at the beginning of the year $\mathrm{J} 2000$, respectively. This is why $\alpha$ and $\delta$ are called equatorial coordinates.

The Cartesian unit vectors along the principal axes of the ICRS are denoted $\boldsymbol{X}, \boldsymbol{Y}, \boldsymbol{Z}$, with $\boldsymbol{Z}$ pointing towards the celestial north pole, $\boldsymbol{X}$ towards the spring equinox and $\boldsymbol{Y}=\boldsymbol{Z} \times \boldsymbol{X}$. 


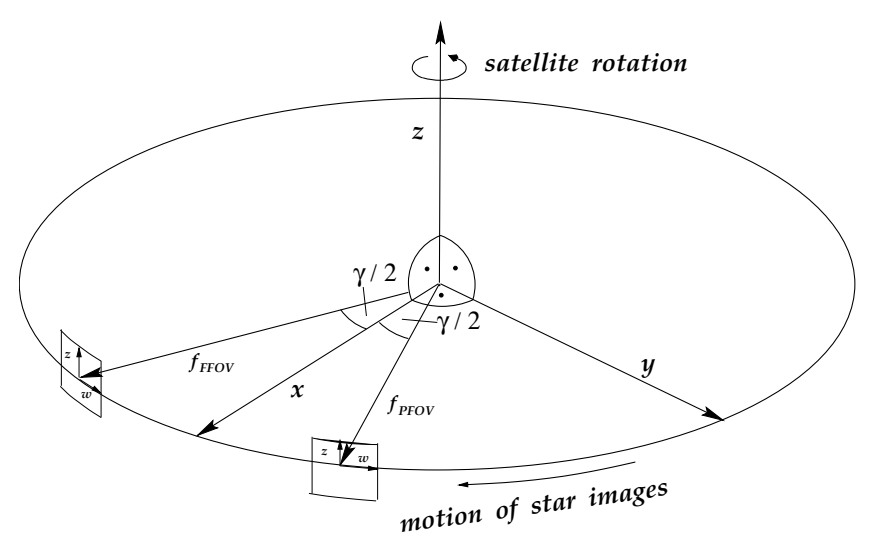

Fig. 1. The satellite system, viewing directions and field-of-view systems. The field-of-view angles $\eta, \zeta$ are not indicated separately; they have the same origin and orientation as $w, z$.

A general unit vector $\boldsymbol{u}$ has direction cosines $X, Y, Z$, such that

$\boldsymbol{u}=X \boldsymbol{X}+Y \boldsymbol{Y}+Z \boldsymbol{Z}$

with $X^{2}+Y^{2}+Z^{2}=1$.

\subsubsection{The satellite system}

For the interpretation of Gaia observations it is useful to express star positions, or more generally, directions in a coordinate system that is rotating with the satellite. This is the satellite system. The unit vectors along the principal axes are called $\boldsymbol{x}$, $\boldsymbol{y}, z$, see Fig. 1. The $z$ axis is the nominal rotation axis of the satellite, with the sun having a positive $z$ coordinate during nominal scientific operations. This means that the $z$ axis points towards Gaia's top covered by the solar arrays. The $x$ axis is in the plane of the two viewing directions (i.e. the two projections of the optical axis of Gaia's Astro telescope onto the sky), bisecting the angle between them, so that it is $\gamma / 2$ away from each of them. The $y$ axis is also in the plane of the two viewing directions such that the system $\boldsymbol{x}, \boldsymbol{y}, \boldsymbol{z}$ is right-handed. A general unit vector $\boldsymbol{u}$ in the satellite system has direction cosines $x, y, z$, such that

$\boldsymbol{u}=x \boldsymbol{x}+y \boldsymbol{y}+z \boldsymbol{z}$

with $x^{2}+y^{2}+z^{2}=1$.

The nominal rotation of Gaia is positive about the positive $z$ axis. The $y$ axis thus precedes the $\boldsymbol{x}$ axis on the sky by 90 degrees.

\subsubsection{Field-of-view coordinates}

In order to describe the actual motion of star images across the focal plane of Gaia, it is useful to define two coordinate systems directly related to the viewing directions, i.e. to the two projections of the optical axis of Gaia's Astro telescope onto the sky. There is one such system per astrometric field of view (thus the name field-of-view coordinates). The two systems differ from the satellite system by a rotation around the $z$ axis by an angle $\pm \gamma / 2$, where $\gamma$ is Gaia's basic angle, i.e. the angle between the two viewing directions.
The meaning of the field coordinates is also shown in Fig. 1. Their origin is at the projection of the optical axis; the $w$ coordinate is reckoned along the nominal scan direction; the $z$ coordinate is orthogonal to it. The orientation is such that star images move towards negative values of $w$, and that celestial objects with a positive $z$ coordinate in the satellite system will have a positive $z$ field-of-view coordinate. In particular, the sun will have a positive $z$ coordinate during nominal scientific operations. This somewhat tricky definition of the orientation of the $z$ field coordinate is necessary because e.g. the physical orientation of the $z$ field coordinate on the focal plane within the satellite is not necessarily parallel to the $z$ axis of the laboratory system.

The unit vectors along the principal axes of either fieldof-view coordinate system are denoted $f, w, z$, with $z$ being identical to the third axis of the satellite system, and

$\boldsymbol{f}=\boldsymbol{x} \cos \gamma / 2 \pm \boldsymbol{y} \sin \gamma / 2=\boldsymbol{x} \cos \gamma / 2+f \boldsymbol{y} \sin \gamma / 2$,
$\boldsymbol{w}=\boldsymbol{y} \cos \gamma / 2 \mp \boldsymbol{x} \sin \gamma / 2=\boldsymbol{y} \cos \gamma / 2-f \boldsymbol{x} \sin \gamma / 2$,

where $f$ is the field-of-view index, which is defined as $f=+1$ for the preceding field of view and $f=-1$ for the following.

The field-of-view coordinates are direction cosines in the field-of-view coordinate system, such that a general unit vector $\boldsymbol{u}$ is

$\boldsymbol{u}=v \boldsymbol{f}+w \boldsymbol{w}+z \boldsymbol{z}$

with $v^{2}+w^{2}+z^{2}=1$

The field-of-view coordinates are related to the coordinates in the satellite system by the simple relations

$$
\begin{aligned}
& v=x \cos \gamma / 2+f y \sin \gamma / 2 ; x=v \cos \gamma / 2-f w \sin \gamma / 2, \\
& w=y \cos \gamma / 2-f x \sin \gamma / 2 ; \quad y=w \cos \gamma / 2+f v \sin \gamma / 2, \\
& z=z
\end{aligned}
$$

\subsubsection{Field-of-view angles}

For some purposes it is more convenient to use field-of-view angles instead of field-of-view coordinates. The field-of-view angles $\eta$ and $\zeta$ are angular longitude and latitude coordinates in the field-of-view systems, connected to the field-of-view coordinates by

$$
\begin{aligned}
v & =\cos \zeta \cos \eta, \\
w & =\cos \zeta \sin \eta, \\
z & =\sin \zeta .
\end{aligned}
$$

In Fig. 1 the field-of-view angles $\eta, \zeta$ are not indicated separately; they have the same origin and orientation as $w, z$.

For clarity and simplicity we will use field-of-view angles rather than field-of-view coordinates in the remainder of this paper.

\subsection{Attitude}

The attitude of the satellite is the orientation of the satellite system with respect to the ICRS. It can expressed by the attitude matrix $A$, an orthonormal $3 \times 3$ matrix. Each line of the matrix contains the equatorial (ICRS) direction cosines of one of the 
satellite system's principal axes. Conversely, the columns of the matrix contain the direction cosines of the ICRS principal axes expressed in the satellite system. Symbolically:

$\mathrm{A}=\left(\begin{array}{l}\boldsymbol{x}^{\prime} \\ \boldsymbol{y}^{\prime} \\ \boldsymbol{z}^{\prime}\end{array}\right)_{\text {ICRS }}=\left(\begin{array}{lll}\boldsymbol{X} & \boldsymbol{Y} & \boldsymbol{Z}\end{array}\right)_{\text {sat.syst. }}$,

where the prime denotes the transpose of a vector.

Multiplying A with any vector $\boldsymbol{u}$ given in ICRS coordinates produces the representation of that vector in the satellite system:

$\left(\begin{array}{l}x \\ y \\ z\end{array}\right)_{u}=\mathrm{A} \cdot\left(\begin{array}{l}X \\ Y \\ Z\end{array}\right)_{u}$

Instead of the rotation matrix A, any other conventional mathematical representation of a three-dimensional rotation can be used, e.g. any set of Euler angles or Tait-Bryan angles, or quaternions.

Note that for a right-handed rotation of the satellite around its positive $z$ axis the star images move towards negative $w$ and $\eta$, see Fig. 1.

\subsection{Simplifications}

For clarity and simplicity it is useful to disregard a number of the actual complexities of the Gaia instruments and operations. They are irrelevant for the fundamental effects to be studied here; and it is easy to re-instate them later on in a full formulation of the problem.

For the present work it is sufficient to consider a single field of view. Thus we set $\gamma=0$, the difference between the satellite system and field-of-view systems vanishes, and application of the attitude directly transforms celestial coordinates into fieldof-view coordinates, resp. angles.

For the present paper it is sufficient to consider a rotation of the satellite around a fixed $z$ axis in space, i.e. the attitude is reduced to a one-dimensional rotation around that axis. For clarity and simplicity we choose to describe it by the third angle of a set of $(3,1,3)$ Euler angles, which usually is denoted by $\psi$ (the first two, usually denoted $\phi$ and $\theta$, define the orientation of the $z$ axis in space). In this model the optical axis of the telescope scans along a great circle, with $\psi$ being a longitude coordinate along that circle. Thus, the rotational motion of the satellite and instrument is described by a function $\psi_{\mathrm{i}}(t)$, where $t$ is some time coordinate and the suffix "i" stands for "instrument".

Furthermore it is useful to describe star positions, or more generally, directions in the astronomical sense in a celestial coordinate system that is oriented along the great circle of the scan. In addition, for the present work we need to consider only the along-scan measurements done by the Gaia instruments. Thus, star positions can simply be described by the along-scan angular coordinate $\psi$ defined above. Let us thus denote a star's celestial coordinate by $\psi_{*}$.

The transformation of a star position to field-of-view angle then takes the extremely simple form

$\eta_{*}(t)=\psi_{*}(t)-\psi_{\mathrm{i}}(t)$.
In actual fact this equation is the definition of attitude (i.e. the equivalent of Eq. (1) in our restricted model). In very simple, mnemonic words: the attitude $\psi_{\mathrm{i}}(t)$ is equal to the longitude coordinate $\psi$ on the sky towards which the telescope $(\eta=0)$ looks at time $t$.

As a further simplification we choose to use the following physical units: angles are given in units of the along-scan size of the CCD pixels (projected back through the telescope optics onto the sky). Time is given in units of the nominal TDI clock interval. The nominal scan speed of the satellite is thus $\omega=$ $\mathrm{d} \psi_{\mathrm{i}}(t) / \mathrm{d} t=1$.

Last but not least we assume that the TDI clocking runs at the nominal pace, with clock stroke number zero taken at the time origin $t=0$. Under this assumption the time $t_{k}$ at which clock stroke $k$ is executed is simply $t_{k}=k$. Let us furthermore define the function $k(t)$ to be the running number $k$ of the last clock stroke executed before (or at) time $t$. Then $k(t)=[t]=$ $\operatorname{INT}(t)$. This assumption, as all others, are taken for clarity and simplicity only. They are not essential for the subject of this paper and can be easily removed in a full formulation of the subject.

\subsection{Observation equation for a transit instrument}

A scanning CCD in TDI mode is a kind of transit instrument. Like the other types of transit instruments mentioned in the introduction it records the transit of optical star images across some fiducial line in the focal plane of the telescope. Roughly speaking, the instant of transit $t_{\text {tr }}$ would be the TDI clock stroke at which a pixel containing the centroid of the image is loaded from the trailing pixel row into the serial read-out register. The role of the fiducial line would then be taken by the centre of that trailing pixel row. Later on we shall see that this concept needs some refinement.

Denoting the location of the trailing pixel row of a CCD chip by its field angle $\eta_{\text {tr }}$ ("tr" stands for "transit"), the observation equation for an along-scan astrometric measurement takes the form already indicated in the introduction:

$\eta_{*}\left(t_{\mathrm{tr}}\right)-\eta_{\mathrm{tr}}=\psi_{*}\left(t_{\mathrm{tr}}\right)-\psi_{\mathrm{i}}\left(t_{\mathrm{tr}}\right)-\eta_{\mathrm{tr}}=0+$ noise.

A more practical form to be used in an actual data reduction process is

$$
\begin{aligned}
\eta_{*}\left(t_{\mathrm{tr}}\right)-\eta_{\text {model }}\left(t_{\mathrm{tr}}\right) & =\eta_{\mathrm{tr}}-\eta_{\text {model }}\left(t_{\mathrm{tr}}\right) \\
& =\Delta \psi_{\mathrm{i}}\left(t_{\mathrm{tr}}\right)+\Delta \psi_{*}\left(t_{\mathrm{tr}}\right)+\Delta \eta_{\mathrm{tr}}+\text { noise }
\end{aligned}
$$

where $\eta_{\text {model }}$ is the field angle of the star computed from the approximately known star position, attitude and instrument calibration, and $\Delta \psi_{\mathrm{i}}\left(t_{\mathrm{tr}}\right), \Delta \psi_{*}\left(t_{\mathrm{tr}}\right), \Delta \eta_{\mathrm{tr}}$ are the sought-for corrections to those approximate values.

The actual segmented form to be used in the Gaia Global Iterative Solution, along with further explanations, can be found in Sect. 1.5 of Lindegren (2003) (Version 3 or higher). A very general form can be found in Chap. 9, Eqs. (102)-(105) of ESA (2000). 


\section{Optical images versus charge images}

In this section we elaborate on the fact that the final image registration in a TDI-operated scanning instrument contains a convolution of the source position, attitude and TDI clocking over the entire CCD exposure time. We analyse this mathematically. From the result of this analysis we proceed to define an effective, astrometric attitude.

\subsection{Physical pixels versus data pixels}

A CCD chip mainly consists of a rectangular array of lightsensitive pixels. This array is finite in both dimensions, e.g. 1966 pixels across scan by 4500 pixels along scan for the Astro CCDs of the Gaia Astro instrument. These light-sensitive pixels will be called physical pixels in the following. The data from a CCD consist of rectangular arrays of photo-electric charges read out from the chip (and digitized for computer storage). The elements of such arrays will be called data pixels in the following. In astronomical applications one finds (charge) images of stars scattered across the data pixel arrays.

In the ordinary (pointed) operational mode of CCDs every exposure yields a data pixel array having the same dimensions as the array of the physical pixels. Each data pixel uniquely corresponds to a specific physical pixel on the chip. Thus a clear conceptual distinction between physical pixels and data pixels does not seem to be necessary in this case.

However, this is different in the case of TDI data. Here, the charge in each data pixel is produced by all physical pixels along one of the CCD's columns. The array size of the data pixels across scan is still the same as that of the physical pixel array (namely the number of columns of the CCD). But along scan the array of data pixels may be "infinitely" long.

Let us introduce a numbering scheme for the individual elements of the TDI data pixel arrays. The index across scan is simply the index of the column of the CCD chip. It does not matter in the following. The index along scan is the running number of the TDI clock stroke at which the pixel was transferred from the trailing CCD pixel row into the read-out register. Let us denote this index by $k$. Note that $k$ corresponds to a time coordinate, not to a spatial location on the focal plane. A star image centered on the trailing CCD pixel row at time $t_{k}$ would be centered on data pixel $k$.

While each photo-electron is located in a particular integer data pixel, the centroid of a recorded star image will (by some centroiding algorithm) be determined as a continuous quantity from the distribution of light (charge) among the data pixels. The centroid of a star image may thus lie in between the pixels. Let us therefore define an along-scan coordinate $\kappa$ as the continuous extension (interpolation) between the integer sequence defined by $k$. This $k$ corresponds to a continuous time coordinate in units of TDI clock strokes.

In the astrometric interpretation of the TDI data stream, $\kappa$ will take the role of $t_{\text {tr }}$ in the observation equation (Eqs. (3) or (4)), and the centre of the trailing pixel row will take the role of the fiducial line at $\eta_{\text {tr }}$.

Under our present assumptions (strictly nominal TDI clocking and $t_{k}=k$ ) the differentiation between $t$ and $\kappa$ may
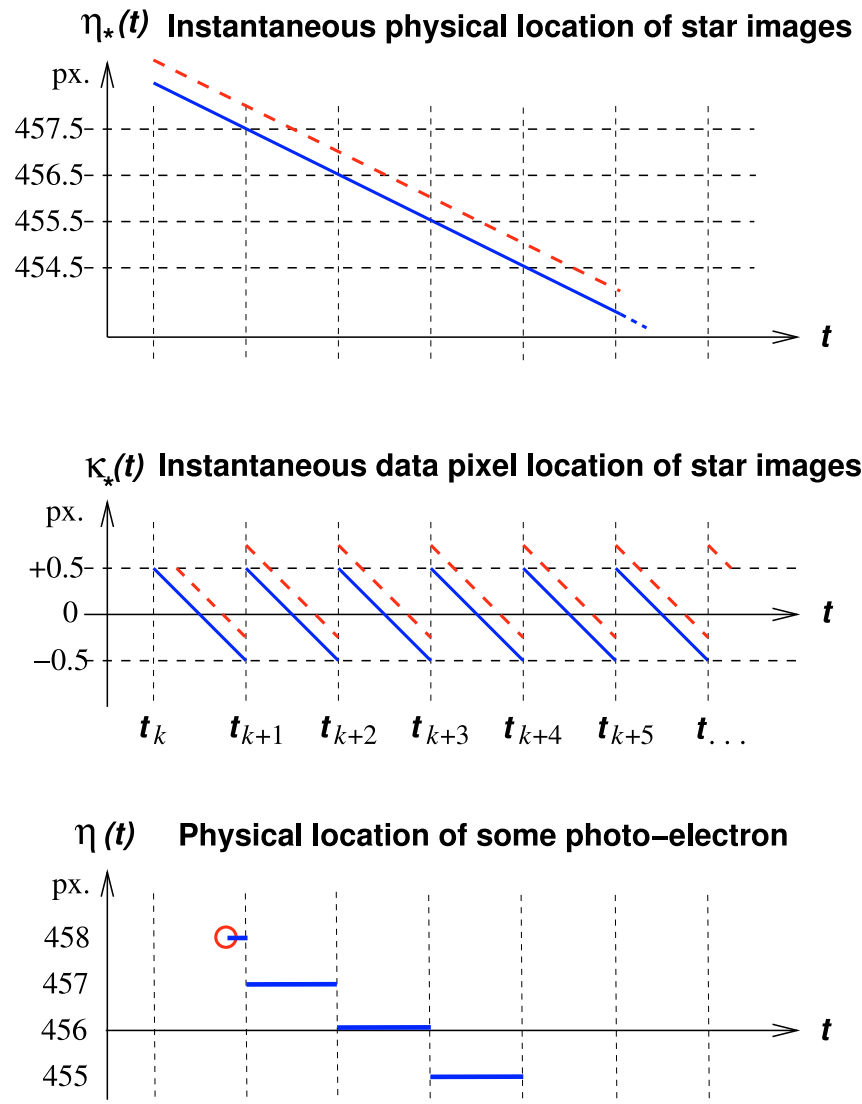

Fig. 2. The locations of optical star images and photo-electrons in physical space and data space. See text.

seem unnecessary and artificial, but it will be important in a practical mission.

\subsection{Data pixel location of a star image}

Let us now try to compute the data pixel location of a star image from first principles. Assume - for the moment - that the telescope rotates at exactly nominal speed, and consider the optical image of a star moving across the focal plane of the scanning telescope, traversing a CCD. Its field-of-view coordinate $\eta_{*}$ decreases with time (see Fig. 2, top panel; the diagonal solid and dashed lines correspond to two stars with slightly different positions $\psi_{*}$ ). Thus we may write, using Eq. (2):

$\eta_{*}(t)=\psi_{*}-\psi_{\mathrm{i}}(t)$

By definition, the TDI clocking shifts the photo-electric charge produced by the moving image downward by one pixel (in physical space, i.e. on the CCD) after each unit of time, so as to keep pace with the optical image ${ }^{1}$. Turning this argument around, we find that in data pixel space the location of the star image is effectively shifted upward by one pixel (see Fig. 2, center panel). We may therefore define an instantaneous data

\footnotetext{
${ }^{1}$ In reality the charge is shifted downwards by a quarter of the physical pixel separation at each clock stroke in the case of Gaia's CCDs. This is an irrelevant detail for the following considerations, except for the numerical values at the end of Appendix A.
} 


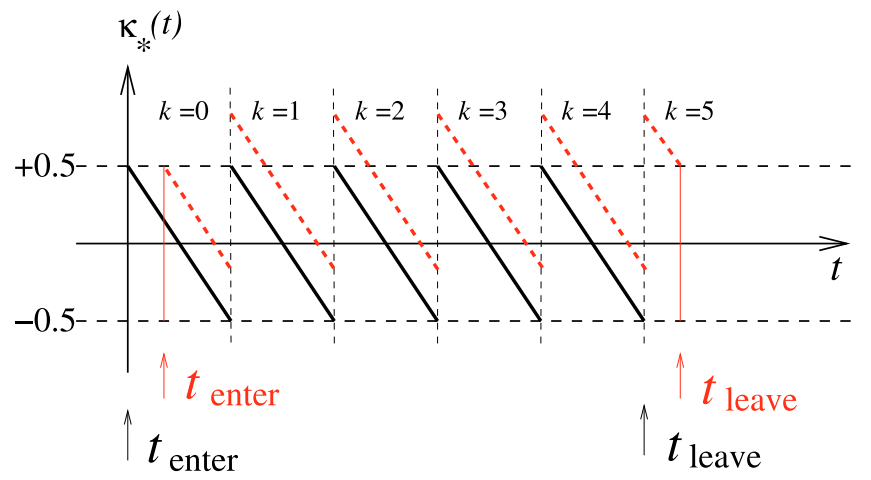

Fig. 3. The data pixel locations of optical star images at nominal scan speed. See text for details.

pixel location of the star image (more precisely: of its optical centroid) as

$\kappa_{*}(t)=\eta_{*}(t)+k(t)+\kappa_{0}=\psi_{*}-\psi_{\mathrm{i}}(t)+k(t)+\kappa_{0}$

as shown in Fig. 2, center panel. Here, $k(t)$ is the running number of the last TDI clock stroke before time $t$ and $\kappa_{0}$ is an initial value of $\kappa$.

The meaning of $\kappa_{0}$ can be illustrated by the following rough consideration: According to Eq. (5) at transit time we have $\kappa_{*}\left(t_{\mathrm{tr}}\right)=t_{\mathrm{tr}}=\eta_{\mathrm{tr}}+k\left(t_{\mathrm{tr}}\right)+\kappa_{0}$. Assuming an integer $t_{\mathrm{tr}}$ we furthermore have $k\left(t_{\mathrm{tr}}\right)=t_{\mathrm{tr}}$, and thus $0=\eta_{\mathrm{tr}}+\kappa_{0}$. In other words: under our present assumptions $\kappa_{0}$ is the negative of the location of the fiducial line.

We can thus rewrite Eq. (5) as

$\kappa_{*}(t)=\psi_{*}-\psi_{\mathrm{i}}(t)-\eta_{\mathrm{tr}}+k(t)=\eta_{*}(t)-\eta_{\mathrm{tr}}+k(t)$.

In a practical mission there would in addition be contributions of $k(0)$ and the basic angle $\pm \gamma / 2$ to $\kappa_{0}$. In any case, $\kappa_{0}$ is an instrumental calibration quantity.

Note that for the solid diagonal lines in the upper and central panels of Fig. 2 the centroid of the star image is arbitrarily assumed to enter the CCD, i.e. to coincide with the leading edge of the first pixel row, exactly at time $t_{k}$ of some TDI clock stroke $k .^{2}$ If the centroid of the star image would enter the CCD at some slightly later time, its instantaneous data pixel location would follow the dashed line.

The bottom panel of Fig. 2 shows, for comparison, the physical location of a particular photo-electron inside the chip. Its time dependence is completely different from both $\eta_{*}(t)$ and $\kappa_{*}(t)$. Here, the electron is assumed to be created in the physical pixel located at $\eta=458$ at some arbitrary instant of time (circle). The data pixel location of the electron is constant in time.

Figure 3 shows an enlargement of the central panel of Fig. 2, Fig. 4 shows the same for the case of a non-nominal (slightly higher) scan speed. In the latter case the instantaneous field angle of the optical image decreases by slightly more than one pixel during each TDI clock interval, yet each TDI shift is only one pixel upwards. Thus, in addition to the saw-tooth pattern of Fig. 3 there is a linear trend in $\kappa_{*}(t)$.

\footnotetext{
2 According to the usual conventions for CCD data, the center of a pixel has integer pixel coordinates; i.e. the edge of a pixel corresponds to the fractional part of the pixel coordinate being 0.5 .
}

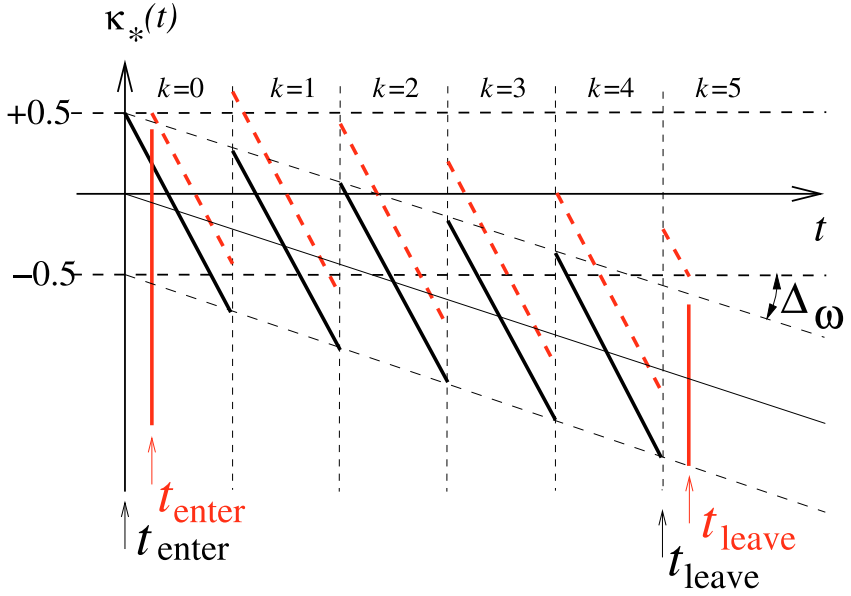

Fig. 4. The data pixel locations of optical star images at non-nominal (higher) scan speed. See text for details.

\subsection{Optical images versus charge images}

The preceding section dealt with instantaneous optical images. However, this is not what is read out from the CCD. Instead, the measured signal consists of the accumulated charge over the entire exposure time. The decisive question in the astrometric interpretation of TDI data is:

What is the data pixel location of this charge image? More precisely: what is the data pixel coordinate $\kappa_{\mathrm{c}}$ of its centroid? ${ }^{3}$

The answer is that the accumulated charge represents the average of the data pixel location of the instantaneous star image over the exposure time. Let us denote the start and end points of the exposure time for a given image as $t_{\text {enter }}$ and $t_{\text {leave }}$, the difference between the two as $t_{\mathrm{exp}}$. Then

$\kappa_{\mathrm{c}}=\frac{1}{t_{\text {exp }}} \int_{t_{\text {enter }}}^{t_{\text {leave }}} \kappa_{*}(t) \mathrm{d} t$.

Inserting Eq. (5) for $\kappa_{*}(t)$ we find

$\kappa_{\mathrm{c}}=\frac{1}{t_{\mathrm{exp}}} \int_{t_{\text {enter }}}^{t_{\text {leave }}}\left[\psi_{*}(t)-\psi_{\mathrm{i}}(t)+k(t)-\eta_{\mathrm{tr}}\right] \mathrm{d} t$.

Assuming $\psi_{*}(t)=$ const over the exposure time, we have

$$
\begin{aligned}
\kappa_{\mathrm{c}} & =\psi_{*}-\eta_{\mathrm{tr}}+\frac{1}{t_{\exp }} \int_{t_{\text {enter }}}^{t_{\text {leave }}}\left[k(t)-\psi_{\mathrm{i}}(t)\right] \mathrm{d} t \\
& =\psi_{*}-\eta_{\mathrm{tr}}+\frac{1}{t_{\exp }} \int_{t_{\text {enter }}}^{t_{\text {leave }}} k(t) \mathrm{d} t-\frac{1}{t_{\exp }} \int_{t_{\text {enter }}}^{t_{\text {leave }}} \psi_{\mathrm{i}}(t) \mathrm{d} t .
\end{aligned}
$$

This is the central equation that will be investigated in the remainder of this paper. Note that - for a given physical attitude $\psi_{\mathrm{i}}(t)$ - the quantity $\kappa_{\mathrm{c}}$ is a function of $\psi_{*}$ only. The integral limits are determined by $\psi_{*}$ via

$\eta_{*}\left(t_{\text {enter }}\right)=\eta_{\text {enter }}=\psi_{*}-\psi_{\mathrm{i}}\left(t_{\text {enter }}\right)$

and

$\eta_{*}\left(t_{\text {leave }}\right)=\eta_{\text {leave }}=\psi_{*}-\psi_{\mathrm{i}}\left(t_{\text {leave }}\right)$,

where $\eta_{\text {enter }}$ and $\eta_{\text {leave }}$ are the field angles of the leading and trailing edges of the CCD, respectively.

3 The suffix "c" stands for either "charge" or "centroid" or both. 
Note furthermore that in general $\kappa_{\mathrm{c}}$ is not equal to $\kappa_{*}\left(t_{\mathrm{tr}}\right)$ !

Equations (7) to (9) assume that the star image enters the CCD at a definite time and suddenly starts to produce photoelectric charge at the full rate. In actual fact the star image is extended, mostly due to the $\frac{\sin x}{x}$ diffraction pattern, and to a lesser extent due to diffusion of light inside the CCD chip. Thus it will gradually start producing charge according to the portion of light flux falling onto the chip. A more general and more precise form of Eq. (7) therefore is

$\kappa_{\mathrm{c}}=\frac{1}{\int_{-\infty}^{+\infty} e(t) \mathrm{d} t} \int_{-\infty}^{+\infty} e(t) \kappa_{*}(t) \mathrm{d} t$,

where $e(t)$ is the "exposure function", i.e. the instantaneous rate (or rather proportion) of star light actually producing photoelectrons at time $t$. Equation (7) in this sense corresponds to a box-shaped exposure function. Note that the realistic exposure function is indeed close to box-shaped, since the width of the CCD is thousands of pixels, while the width of the image is just a few pixels.

The exposure function can carry more information than just the shape of the optical image: a non-zero CTI (i.e. a loss of part of the generated charges during the TDI transport) would be represented by a slope of the flat "roof" portion of $e(t)$; a blocked CCD column would be represented by a one-sided cutaway portion of $e(t)$.

The term $e(t)$ in Eq. (10) is an abbreviated notation used for clarity. In that notation each star image would have a different exposure function. In actual fact, all exposure functions have (almost) the same shape, but are shifted in time according to $t_{\text {enter }}$ for each star. So, a more complete notation would be $e\left(t-t_{\text {enter }}\right)$ in both integrals of Eq. (10). For a non-nominal scan speed, one would have $e\left(\omega t-\omega t_{\text {enter }}\right)$ instead. And for a non-uniform (i.e. realistic) CCD chip one would have one such function for each CCD column.

\subsection{Astrometric attitude}

Section 3.3 has clarified that $\kappa_{\mathrm{c}}\left(\psi_{*}\right)$ is the primary astrometric observable of Gaia. The basic task of the astrometric data reduction is to derive the star position $\psi_{*}$ from $\kappa_{\mathrm{c}}$. Equation (9) tells us that this is only possible when at the same time we derive some calibration and attitude information.

In Eq. (9) the primary observable $\kappa_{\mathrm{c}}$ is not determined by the physical attitude at some transit time. Instead, it contains an average (running mean) of the physical attitude over an extended interval of time. It is mathematically impossible to reconstruct a general function from a running mean. This means that the physical attitude cannot be derived from the CCD data. It is strictly unobservable.

If, in spite of this complication, we still want to regard the scanning CCD as a transit instrument, i.e. if we want to use observation equations of the form of Eq. (3) (or (4)), we have no choice but to replace the (unobservable) quantities contained in Eq. (3) (and (4)) by the actual observables of the TDI measuring process. This leads to the definition of an effective or apparent astrometric attitude. For brevity we call it astrometric attitude in the following. In analogy with $\kappa_{\mathrm{c}}-$ and to distinguish it from the physical instrument attitude $\psi_{\mathrm{i}}$ - we denote it as $\psi_{\mathrm{c}}$, where the suffix "c" may stand for either "charge" or "centroid" or both.

Consider a star having along-scan coordinate $\psi_{*}$ and observed data pixel coordinate $\kappa_{\mathrm{c}}\left(\psi_{*}\right)$. Then we identify $\kappa_{\mathrm{c}}$ as the observable transit time $t_{\text {tr }}$ and simply define the astrometric attitude $\psi_{\mathrm{c}}\left(t_{\mathrm{tr}}\right)$ as function of $t_{\mathrm{tr}}$ by

$\psi_{\mathrm{c}}\left(\kappa_{\mathrm{c}}\right)=\psi_{\mathrm{c}}\left(t_{\mathrm{tr}}\right)=\psi_{*}-\eta_{\mathrm{tr}}$

in analogy with Eq. (2).

Assume that $\eta_{\mathrm{tr}}=0$ (this is not a restriction on the generality of our considerations, since this can always be achieved in practice by a simple shift of the $\eta$ origin on the focal plane), then we have:

$\psi_{\mathrm{c}}\left(t_{\mathrm{tr}}\right)=\psi_{\mathrm{c}}\left(\kappa_{\mathrm{c}}\left(\psi_{*}\right)\right)=\psi_{*}$

So the astrometric attitude is defined as the inverse function of $\kappa_{\mathrm{c}}\left(\psi_{*}\right)$. This may be an un-intuitive concept, but it was unknowingly implied by all past descriptions of Gaia astrometry, both in publications and in technical documents.

The redefined observation equation then reads

$\eta_{*}\left(t_{\mathrm{tr}}\right)-\eta_{\mathrm{tr}}=\psi_{*}-\psi_{\mathrm{c}}\left(\kappa_{\mathrm{c}}\right)-\eta_{\mathrm{tr}}=0+$ noise

in analogy to Eq. (3), and the more practical form in analogy to Eq. (4) becomes

$\eta_{\mathrm{tr}}-\eta_{\text {model }}\left(\kappa_{\mathrm{c}}\right)=\Delta \psi_{\mathrm{i}}\left(\kappa_{\mathrm{c}}\right)+\Delta \psi_{*}+\Delta \eta_{\mathrm{tr}}+$ noise

\section{Astrometric consequences I: Attitude modelling}

The analysis of Gaia's observation process introduced in Sect. 3 has some important consequences. The concept of astrometric attitude is so un-intuitive that its consequences can only be illustrated in a few idealized cases. However the main results are fully valid in more general cases as well.

In Appendix A we will demonstrate that for sufficiently large $N_{\mathrm{px}}$, and for scan speeds not too far from nominal, the discreteness of the charge shifting does not cause any important effects (in fact there is a TDI-induced periodic jitter, but it will be shown to be negligibly small). So let us make another approximation and assume continuous shifting of the charges, i.e. very tiny pixels. Again, this approximation is for illustration purposes only. The complete algebra with the discrete shifting over a finite number of pixels will be given in Appendix A.

\subsection{Constant, nominal scan speed}

Let us take the definition of $\kappa_{\mathrm{c}}$, Eq. (9), and identify $\eta_{\text {tr }}$ with the trailing edge $\eta_{\text {leave }}$ of the trailing CCD pixel. Then

$\kappa_{\mathrm{c}}=\psi_{*}-\eta_{\text {leave }}+\frac{1}{t_{\text {exp }}} \int_{t_{\text {enter }}}^{t_{\text {leave }}}\left[k(t)-\psi_{\mathrm{i}}(t)\right] \mathrm{d} t$.

With continuous shifting of charge, i.e. $k(t)=t$ we have

$\kappa_{\mathrm{c}}=\psi_{*}-\eta_{\text {leave }}+\frac{1}{t_{\text {exp }}} \int_{t_{\text {enter }}}^{t_{\text {leave }}}\left[t-\psi_{\mathrm{i}}(t)\right] \mathrm{d} t$ 
With constant, nominal scan speed, i.e. $\psi_{\mathrm{i}}(t)=\psi_{0}+t$ we have

$$
\begin{aligned}
\kappa_{\mathrm{c}} & =\psi_{*}-\eta_{\text {leave }}+\frac{1}{t_{\text {exp }}} \int_{t_{\text {enter }}}^{t_{\text {leave }}}\left[t-t-\psi_{0}\right] \mathrm{d} t \\
& =\psi_{*}-\eta_{\text {leave }}-\frac{1}{t_{\text {exp }}} \psi_{0}\left[t_{\text {leave }}-t_{\text {enter }}\right] \\
& =\psi_{*}-\eta_{\text {leave }}-\psi_{0} .
\end{aligned}
$$

With the definition of the astrometric attitude $\psi_{\mathrm{c}}$ being the inverse function of $\kappa_{\mathrm{c}}$, i.e. with Eq. (11), we thus have (after simply solving the previous equation for $\psi_{*}$ and inserting into Eq. (11))

$$
\psi_{\mathrm{c}}\left(\kappa_{\mathrm{c}}\right)=\psi_{*}-\eta_{\text {leave }}=\kappa_{\mathrm{c}}+\psi_{0}=\psi_{\mathrm{i}}\left(\kappa_{\mathrm{c}}\right) .
$$

In other words: at strictly nominal scan speed the physical and the astrometric attitude are identical. This seemingly trivial result nothing but shows that we have developed a reasonable overall concept. In the simplest possible case, the astrometric attitude (in properly scaled data space) is identical to the physical attitude (in real space).

\subsection{Constant, non-nominal scan speed}

We again start with Eq. (9), introducing continuous shifting of charge $(k(t)=t)$ and physical attitude $\psi_{\mathrm{i}}(t)=\psi_{0}+\left(1+\Delta_{\omega}\right) t$ to find

$$
\begin{aligned}
\kappa_{\mathrm{c}} & =\psi_{*}-\eta_{\text {leave }}+\frac{1}{t_{\text {exp }}} \int_{t_{\text {enter }}}^{t_{\text {leave }}}\left[t-\left(1+\Delta_{\omega}\right) t-\psi_{0}\right] \mathrm{d} t \\
& =\psi_{*}-\eta_{\text {leave }}+\frac{1}{t_{\text {exp }}} \int_{t_{\text {enter }}}^{t_{\text {leave }}}\left[-\Delta_{\omega} t-\psi_{0}\right] \mathrm{d} t \\
& =\psi_{*}-\eta_{\text {leave }}-\psi_{0}-\frac{\Delta_{\omega}}{t_{\text {exp }}} \int_{t_{\text {enter }}}^{t_{\text {leave }}} t \mathrm{~d} t \\
& =\psi_{*}-\eta_{\text {leave }}-\psi_{0}-\frac{\Delta_{\omega}}{t_{\text {exp }}}\left[\frac{1}{2} t_{\text {leave }}^{2}-\frac{1}{2} t_{\text {enter }}^{2}\right] \\
& =\psi_{*}-\eta_{\text {leave }}-\psi_{0}-\frac{\Delta_{\omega}}{2 t_{\text {exp }}}\left[t_{\text {leave }}^{2}-\left(t_{\text {leave }}-t_{\text {exp }}\right)^{2}\right] \\
& =\psi_{*}-\eta_{\text {leave }}-\psi_{0}-\frac{\Delta_{\omega}}{2 t_{\text {exp }}}\left[2 t_{\text {leave }} t_{\text {exp }}-t_{\text {exp }}^{2}\right] \\
& =\psi_{*}-\eta_{\text {leave }}-\psi_{0}-\Delta_{\omega}\left[t_{\text {leave }}-\frac{1}{2} t_{\text {exp }}\right] .
\end{aligned}
$$

Before we can evaluate this further we have to compute $t_{\text {leave }}$ and $t_{\text {exp }}$. We denote the field angles of the leading and trailing edges of the CCD by $\eta_{\text {enter }}$ and $\eta_{\text {leave }}$, respectively. We furthermore note that the difference between the two - in the case of real, extended pixels - is equal to the number $N_{\mathrm{px}}$ of pixel rows along scan, which in turn is equal to the exposure time at nominal scan speed $\omega=1$. For clarity we denote this difference by $N_{\mathrm{px}}$, although the assumption of continuous charge shifting implicitly means infinitely many, infinitely small pixels. Then:

$$
\begin{aligned}
t_{\text {leave }} & =\frac{\psi_{*}-\psi_{0}-\eta_{\text {leave }}}{1+\Delta_{\omega}}, \\
t_{\text {enter }} & =\frac{\psi_{*}-\psi_{0}-\eta_{\text {enter }}}{1+\Delta_{\omega}}=\frac{\psi_{*}-\psi_{0}-\eta_{\text {leave }}-N_{\mathrm{px}}}{1+\Delta_{\omega}}, \\
t_{\text {exp }} & =\frac{N_{\mathrm{px}}}{1+\Delta_{\omega}} .
\end{aligned}
$$

Inserting these values into Eq. (12) yields

$$
\begin{aligned}
\kappa_{\mathrm{c}}= & \psi_{*}-\eta_{\text {leave }}-\psi_{0}-\Delta_{\omega}\left(\frac{\psi_{*}-\eta_{\text {leave }}-\psi_{0}}{1+\Delta_{\omega}}\right)+\frac{\Delta_{\omega}}{2} \frac{N_{\mathrm{px}}}{1+\Delta_{\omega}} \\
= & \psi_{*}\left(1-\frac{\Delta_{\omega}}{1+\Delta_{\omega}}\right)-\eta_{\text {leave }}\left(1-\frac{\Delta_{\omega}}{1+\Delta_{\omega}}\right) \\
& -\psi_{0}\left(1-\frac{\Delta_{\omega}}{1+\Delta_{\omega}}\right)+\frac{\Delta_{\omega}}{2} \frac{N_{\mathrm{px}}}{1+\Delta_{\omega}} .
\end{aligned}
$$

Noting that $1-\frac{\Delta_{\omega}}{1+\Delta_{\omega}}=\frac{1}{1+\Delta_{\omega}}$ we find

$\kappa_{\mathrm{c}}=\psi_{*} \frac{1}{1+\Delta_{\omega}}-\eta_{\text {leave }} \frac{1}{1+\Delta_{\omega}}-\psi_{0} \frac{1}{1+\Delta_{\omega}}+\frac{\Delta_{\omega}}{2} \frac{N_{\mathrm{px}}}{1+\Delta_{\omega}}$.

As in the previous section we can form the inverse function by solving this equation for $\psi_{*}$ to find

$\psi_{*}=\left(1+\Delta_{\omega}\right) \kappa_{\mathrm{c}}+\eta_{\text {leave }}+\psi_{0}-\Delta_{\omega} \frac{N_{\mathrm{px}}}{2}$

and with Eq. (11):

$$
\begin{aligned}
\psi_{\mathrm{c}}\left(\kappa_{\mathrm{c}}\right) & =\psi_{*}-\eta_{\text {leave }}=\left(1+\Delta_{\omega}\right) \kappa_{\mathrm{c}}+\psi_{0}-\Delta_{\omega} \frac{N_{\mathrm{px}}}{2} \\
& =\psi_{\mathrm{i}}\left(\kappa_{\mathrm{c}}\right)-\Delta_{\omega} \frac{N_{\mathrm{px}}}{2} \neq \psi_{\mathrm{i}}\left(\kappa_{\mathrm{c}}\right) .
\end{aligned}
$$

In this case the physical and the astrometric attitude are no longer identical! From Eqs. (13) and (14) we:

$$
\begin{aligned}
\psi_{\mathrm{c}}\left(\kappa_{\mathrm{c}}\right) & =\left(1+\Delta_{\omega}\right) \kappa_{\mathrm{c}}+\psi_{0}-\left(1+\Delta_{\omega}\right) \Delta_{\omega} \frac{N_{\mathrm{px}}}{2\left(1+\Delta_{\omega}\right)} \\
& =\psi_{\mathrm{i}}\left(\kappa_{\mathrm{c}}-\Delta_{\omega} \frac{N_{\mathrm{px}}}{2\left(1+\Delta_{\omega}\right)}\right) \\
& =\psi_{\mathrm{i}}\left(\kappa_{\mathrm{c}}-\Delta_{\omega} \frac{t_{\exp }}{2}\right) .
\end{aligned}
$$

In other words: for a constant satellite rotation rate that differs from the nominal one by $\Delta_{\omega}$ the astrometric attitude is a retarded version of the physical attitude - retarded by $\Delta_{\omega} \frac{t_{\text {exp }}}{2}$.

\subsection{A sudden jump in scan speed}

We briefly explain how a sudden jump in physical scan speed ${ }^{4}$ would appear in the astrometric attitude. The algebra is lengthy but simple, as in the previous two cases. Knowing the outcome of the previous two sections, the result is quite easy to understand.

Let us assume that before the jump we have nominal scan speed. It is obvious that some time before and some time after the sudden jump in scan speed we have the situations described in the previous two sections. This is illustrated by the left part and by the straight portions of the two lower bold lines in the right part of Fig. 5. In between there must of course be some transition between the two situations. In the representation of Fig. 5 that transition takes the shape of a parabola.

In mathematical terms: A sudden discontinuity in the first derivative of the physical attitude (i.e. in the scan rate) is

\footnotetext{
4 This could be affected by a micro-meteoroid hit or by an attitude control gas jet firing.
} 


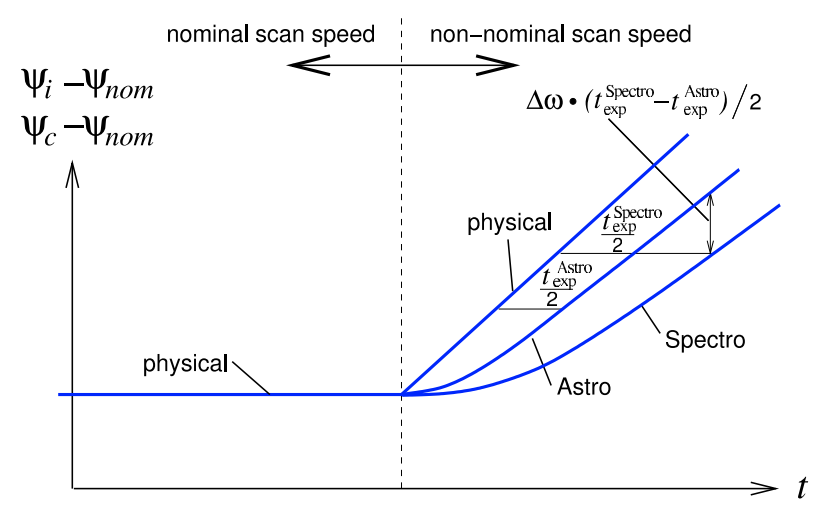

Fig. 5. Physical attitude $\psi_{\mathrm{i}}$ and astrometric attitude $\psi_{\mathrm{c}}$ as functions of time. For clarity and ease of presentation, the difference to the nominal attitude $\psi_{\text {nom }}$ is plotted, where $\psi_{\text {nom }}$ is the attitude for constant, nominal scan rate. At some instant of time (marked by the vertical dashed line) a sudden change in the physical scan rate is assumed. For more explanations see text.

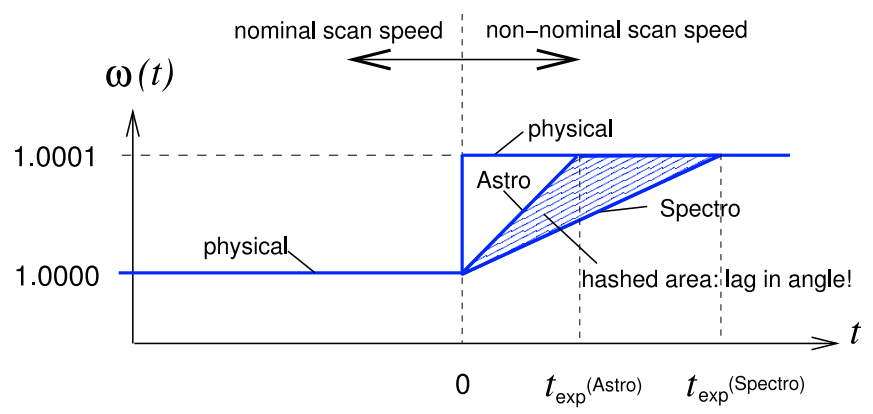

Fig. 6. Scan rates, i.e. time derivatives of the physical attitude $\psi_{\mathrm{i}}$ and astrometric attitude $\psi_{\mathrm{c}}$ as functions of time. As for Fig. 5 a sudden change in the physical scan rate is assumed at the instant of time marked by the vertical dashed line. For more explanations see text.

smeared into two separate discontinuities in the second derivative of the astrometric attitude. The two discontinuities have different signs, are separated by $t_{\exp }$ and have absolute values of $\pm \Delta \omega / t_{\exp }$. The net result is that, as derived in the previous section, the astrometric attitude eventually "lags" behind the physical attitude by $t_{\exp } / 2$ in time, and by $\Delta_{\omega} t_{\exp } / 2$ in angle.

An even more graphic and plausible presentation of the same fact is given in Fig. 6 by plotting the derivative of the attitude instead of its difference to the nominal. Charges read out from the CCD before the instant of the discontinuity do not know anything about the future. Thus the astrometric attitude derived from them represents the nominal scan speed (left). Charges read out more than $t_{\text {exp }}$ after the discontinuity have "seen" only the increased scan rate. Thus the astrometric attitude derived from them represents the increased scan speed after the jump (right). Charges read out $t_{\exp } / 2$ after the jump have experienced the nominal scan speed over half of the exposure time, and the increased one over the other half. Thus the astrometric attitude derived from them indicates a scan speed halfway between the two other values.

The character of the astrometric attitude for more general cases is easy to deduce from the above considerations: it is a smoothed and somewhat "retarded" average over the physical attitude. A slow sinusoidal variation of the physical scan speed, for instance, would be transformed into a phase-shifted sinusoidal with a very slightly reduced amplitude. For a quick sinusoidal variation the remaining amplitude will strongly depend on the precise frequency (being zero if the exposure time is an integer multiple of the period of scan speed variation), while the phase shift always remains at the equivalent of $t_{\exp } / 2$.

\subsection{Different attitudes in different instruments}

As already indicated in Figs. 5 and 6 the astrometric attitudes are different for different Gaia instruments, because their exposure times are different. They would be identical if and only if the physical scan speed would be strictly constant over the whole mission (which it is not). This is the most important finding of this paper. It is also the one with the greatest astrometric effect.

That the effect is very big will be demonstrated presently. But more importantly, it is easy to show that the difference between the astrometric attitudes of different instruments for a real (unknown) physical attitude is highly non-trivial (in contrast to the impression given by the idealized case discussed in the previous section). In Appendix B we prove that even in the absence of any noise it is mathematically impossible to compute the astrometric Spectro attitude from the astrometric Astro attitude, or vice versa.

However, the true meaning of the quantity $\Delta_{\omega}$ introduced in Sect. 4.2 is not a deviation of the actual physical scan speed from the nominal one, but a deviation of the TDI clock rate from the actual physical scan rate. Thus, the newly discovered "attitude lag" effect would disappear if one would use the correct TDI clock rate at all times. But such deviations between the TDI rate and the physical scan rate are unavoidable in practice. There is no way to obtain a sufficiently precise real-time knowledge of the physical scan rate ${ }^{5}$. Furthermore, such deviations are perfectly acceptable for the mission. If they are kept within reasonable limits they will not have any significant effect on the astrometric precision, nor on the photometric precision or the angular resolution of the instruments.

In the Gaia astrometric instruments and photometers, the core peaks of the optical point-spread functions have a diameter of four or five pixels. An unavoidable TDI smear of half a pixel ${ }^{6}$ is added to this, due to the saw-tooth pattern in Fig. 3. As long as the deviation between the TDI rate and the physical scan rate does not cause more than, say, half a pixel of additional image smear, the images are not significantly altered.

To determine on the size of the "attitude lag" effect, let us assume a (practically negligible) 0.1-pixel smear over the Astro CCDs with 4500 pixel rows along scan. Thus $\Delta_{\omega}=$ $60^{\prime \prime} / \mathrm{s} / 10 / 4500=1.33 \mathrm{mas} / \mathrm{s}$. This (not quite incidentally) is the typical peak-to-valley variation of the "absolute rate error" for Gaia from the attitude simulations presented in

\footnotetext{
5 This was somewhat different for Hipparcos. There the sky mappers yielded an independent scan velocity estimate of sufficient precision. For Gaia this would necessitate centroiding and calibration of sky mapper images to $10^{-3}$ Airy diameters, which is unrealistic because these images are not well sampled.

${ }^{6}$ More precisely half a quarter pixel, see Footnote 1.
} 
Table 1. The size of the "attitude lag" effect of various Gaia instruments with respect to Astro, the main astrometric instrument. The assumed numerical value for $\Delta_{\omega}$ is $1.33 \mathrm{mas} / \mathrm{s}$, as explained in the text. The abbreviation "St. Tr." means the Gaia star trackers, assumed to have essentially zero exposure time.

\begin{tabular}{cccc}
\hline \hline Instrument & $t_{\exp }$ & $\left|\Delta t_{\exp } / 2\right|$ & $\Delta \omega\left|\Delta t_{\exp } / 2\right|$ \\
\hline Astro & $3.3 \mathrm{~s}$ & - & - \\
Spectro & $12.0 \mathrm{~s}$ & $4.3 \mathrm{~s}$ & $5720 \mu \mathrm{as}$ \\
St.Tr. & $0.1 \mathrm{~s}$ & $1.6 \mathrm{~s}$ & $2130 \mu \mathrm{as}$ \\
\hline Astro, gate 11 & $1.5 \mathrm{~s}$ & $0.9 \mathrm{~s}$ & $1200 \mu \mathrm{as}$ \\
Astro, gate 9 & $0.38 \mathrm{~s}$ & $1.46 \mathrm{~s}$ & $1940 \mu \mathrm{as}$ \\
Astro, gate 3 & $0.006 \mathrm{~s}$ & $1.65 \mathrm{~s}$ & $2200 \mu \mathrm{as}$ \\
\hline
\end{tabular}

Fig. 3.5.5-A of the Final Report on the Gaia System Level Technical Reassessment Study (Astrium 2002).

Table 1 presents the relevant numerical values for the various Gaia instruments, along with the resulting size of the "attitude lag" effect with respect to the astrometric attitude derived from Astro measurements.

Thus, if one would naively use the high-precision astrometric attitude from Astro for the astrometric interpretation of Spectro measurements, discrepancies of the order of several milli-arcseconds would arise. Since these discrepancies would vary with time (in parallel to the time variations of $\Delta_{\omega}$ ), but would at any time be identical for all simultaneously observed stars, one would interpret them as a time dependence of the geometric calibration of Spectro. In other words: the Spectro CCDs would apparently "float around" on the focalplane assembly by several milli-arcseconds on timescales of the order of $50 \mathrm{~s}$ (see the already mentioned Fig. 3.5.5-A of the Final Report on the Gaia System Level Technical Reassessment Study, Astrium 2002).

This is not just a funny oddity, but would have real scientific drawbacks if unaccounted for. This will be discussed in Sects. 4.5 and 6.

Note that the biggest number in Table 1 has no scientific effect. In the Spectro a shift of 5.7 mas corresponds to only 0.02 "Airy" radii of the optical point-spread function, or a shift of $0.08 \mathrm{~km} \mathrm{~s}^{-1}$ in radial velocity.

\subsection{Separate astrometric reductions for different instruments?}

The statement that it is mathematically impossible to compute the astrometric Spectro attitude from the astrometric Astro attitude (or vice versa) leads to the question: would it thus be necessary to perform separate astrometric reductions for different Gaia instruments?

The answer is no. First of all, the source parameters and the global parameters are independent of the instrument (except for the unresolved binaries). Thus the steps "source processing" and "global processing" of Gaia's Global Iterative Solution (GIS, see ESA 2000, Sect. 9.5, and Lindegren 2001) need not be repeated. Separate calibration processing is necessary anyway, regardless of the "attitude lag" effect. Consequently, that effect would at most force separate attitude processings - if no better solution could be found.
An approximate solution is suggested by Fig. 5: if $\Delta_{\omega}$ is sufficiently constant over time intervals longer than the relevant exposure times, we can use Eqs. (15) or (16) to correct for the "attitude lag" effect. More quantitatively, after such a correction the remaining error would clearly be of the order of $\left(\Delta t_{\exp } / 2\right)^{2} \mathrm{~d} \Delta_{\omega} / \mathrm{d} t$. Again using the characteristics of the physical attitude indicated by the afore-mentioned simulations (specifically: assuming a quasi-sinusoidal variation of $\Delta_{\omega}$ with a peak-to-peak amplitude of $1.33 \mathrm{mas} / \mathrm{s}$ and a typical period of $100 \mathrm{~s}$ ) the remaining error would be of the order of $770 \mu$ as for the correction from Astro to Spectro. This is close to negligible. In the case of the correction from Astro to Astro gate No. 3, the remaining error would be of the order of $110 \mu \mathrm{as}$. This is of the same order of magnitude as the pixel-periodic jitter effect (see Sect. 6) and much larger than what is envisaged as measurement precision for bright stars.

This means that in the case of the Spectro instrument an extremely simple a-posteriori correction could reduce the problem to acceptable levels - if the physical attitude is sufficiently smooth on timescales of a few exposure times. Note that the amplitude of the variations in physical scan rate enters the remaining error only linearly, while their frequency enters quadratically. The actual quality of the simple correction will have to be investigated further, using more detailed characteristics of Gaia's expected physical attitude. It may well turn out that separate attitude reconstructions are indeed necessary for the different instruments. However, the rough estimate in the preceding paragraph makes this appear not very likely.

\section{Astrometric consequences II: Source modelling}

Throughout most of Sects. 3 and 4 we have assumed that the proper direction to the observed sources (represented by $\psi_{*}$ ) does not change over the exposure time. In reality, the first term in the central equation investigated in this paper (Eq. (9)) is an integral over the exposure time as well. From Eq. (8) it is obvious that the true meaning of $\psi_{*}$ in all equations of Sects. 3 and 4 is the average of $\psi_{*}(t)$ over the exposure time.

For all objects with sufficiently small (and/or sufficiently smooth) changes of proper direction over the exposure time, this can be approximated by $\psi_{*}\left(t_{\mathrm{tr}}-t_{\exp } / 2\right)$.

More precisely, and following Eq. (10), the effective time of observation is

$t_{\mathrm{obs}}=\frac{1}{\int_{-\infty}^{+\infty} e(t) \mathrm{d} t} \int_{-\infty}^{+\infty} e(t) t \mathrm{~d} t$.

Using $\psi_{*}\left(t_{\mathrm{tr}}\right)$ instead of $\psi_{*}\left(t_{\mathrm{obs}}\right)$ would cause particularly strong astrometric discrepancies in the case of solar-system objects. A typical minor planet observed by Gaia has an apparent motion of the order of $0.5^{\prime} / \mathrm{h}$ to $1^{\prime} / \mathrm{h}$, i.e. $8 \mathrm{mas} / \mathrm{s}$ to $17 \mathrm{mas} / \mathrm{s}$.

The effect is also relevant for stars. For Gaia the aberration of star light changes by up to $4 \mu \mathrm{as} / \mathrm{s}$ due to the heliocentric acceleration of the spacecraft (for a geocentric orbit the effect is two orders of magnitude greater). Neglect of this would cause a quasi-sinusoidal variation of Gaia's basic angle with the period of the spacecraft rotation and an amplitude of up to about $6 \mu$ as (for the Astro instrument). 
There is an interesting consequence of Eqs. (17) and (16): Both the effective $\psi_{*}$ (from Eq. (17)) as well as the "attitude lag" effect (from Eq. (16)) will be changed if there is a blocked CCD column. This means:

1. Blocked CCD columns will have a very noticeable systematic astrometric effect.

2. This effect can be used to find the along-scan location of the block on the chip.

\section{Astrometric consequences III: CCD gates}

The results of the preceding two sections are of particular importance for the reduction of bright stars with Gaia if CCD gates are be used for their measurement. The gates will lead to very short exposure times for bright stars. Thus their actual time of observation will be very close to the readout instant, and the astrometric attitude that they "see" will include much more short-period noise than that for the fainter stars.

There is a further effect of the gates: if the gates are to be used to acquire an initial attitude, the "attitude lag" effect will be largely absent from that attitude. It will appear, however, as soon as the full Astro CCDs are used in the operational highprecision real-time attitude control scheme. We guess that this has no practical consequences, but may be relevant for Gaia's attitude control system.

\section{Photometric consequences: Source modelling}

All that in Sect. 5 was said about source position of course holds for source brightness as well. The effective time of observation for Gaia's photometry is not $t_{\mathrm{tr}}$ but half an exposure time (i.e. up to a few seconds) earlier. For a photometrist, who is used to long exposures and, at the same time, rapid changes of his/her source parameters, this is a trivial statement. It is added here for completeness only. With the short exposure times of Gaia the difference between $t_{\text {tr }}$ and the mean time of observation will be photometrically relevant for very few sources, if any.

\section{Discussion and conclusion}

\subsection{General}

We have found a mathematically strict definition of an astrometric attitude for observations done in TDI mode. This definition

- retains the formalism of a transit instrument and puts it on a firm mathematical and physical basis;

- reflects the time-averaging nature of the measurement process;

- uses only the actual observables from the TDI output.
Any alternative definition of the attitude would not remove the effects discussed in Sects. 4.4 and 4.5, but would only make them more obscure. The attitudes "seen" by instruments with different exposure times are intrinsically different, because they are averages over different time intervals. This cannot be eliminated, as shown in Appendix B.

However, in practice this will not be a problem, as argued in Sect. 4.5: a very simple approximate transformation between different instruments will normally yield sufficiently high precision. Only very repid changes (discontinuities) of the scan rate may have a practical effect.

Similarly, the consequences of our analysis on source modelling (Sects. 5 to 7) are important in size, but are trivial to implement.

\subsection{Practical consequences for Gaia}

The practical consequences for the specific case of Gaia can be summarised as follows:

- The traditional formulation of the observation equations can be kept.

- The traditional formulation of the data reduction, especially of attitude determination, can be kept.

- The time lag effect must be taken into account when instruments of different exposure times are involved. In the present Gaia design this is relevant only for observations of bright stars using CCD gates.

- The source modelling must take into account the difference between the effective time of observation and readout time. This is especially important in the astrometry of asteroids and in the photometry of rapidly variable stars.

- All this must be included in the Gaia system simulator and in the Gaia data reduction.

- For bright stars observed with gates, three problems occur: reduced attitude smoothing, large time-lag effect and reduced averaging-out of CCD irregularities (pixel-to-pixel variations). This may argue in favour of bright-star astrometry using diffraction spikes rather than using the gates.

\section{References}

Astrium 2002, in Final Report on the Gaia System Level Technical Reassessment Study, Document Ref. EF5/FR/PC/038.02, Astrium, 2002-06-27

ESA 2000, in Gaia Concept and Technology Study Report, ESA-SCI $(2000) 4$

ESA 2005, in The Three-Dimensional Universe with Gaia, ESA SP-576, Proc. of a conference held at Obs. de Paris-Meudon, France, Oct. 4-7, 2004, ed. C. Turon, K. O’Flaherty, \& M. A. C. Perryman, in press

Lindegren, L. 2001, Gaia Technical Document GAIA-LL-034

Lindegren, L. 2003, Gaia Technical Document GAIA-LL-044 
U. Bastian and M. Biermann: Astrometric meaning and interpretation of high-precision TDI CCD data, Online Material p 1

\section{Online Material}


U. Bastian and M. Biermann: Astrometric meaning and interpretation of high-precision TDI CCD data, Online Material p 2
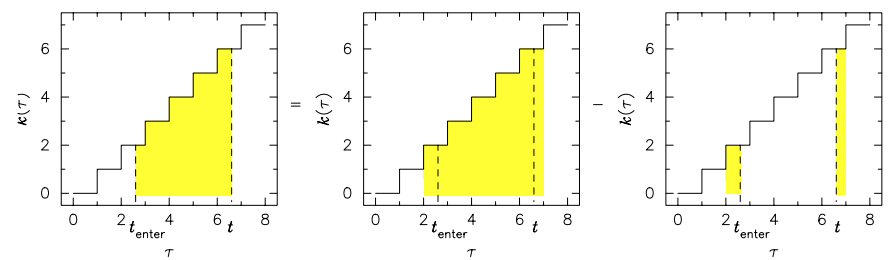

Fig. A.1. The computation of the integral of the step function between $t_{\text {enter }}$ and $t_{\text {leave }}$; this integral equals the difference of the two areas on the right-hand side of the sketch.

\section{Appendix A: Detailed algebra}

In this section we will demonstrate that for scan speeds that will not deviate too much from the nominal one there is a TDIinduced periodic jitter in the case of incorrect clocking and a non-integer exposure time (and only in this case). It also will be shown that this effect is negligible for typical CCD sizes of a few thousand pixels. Unlike in Sect. 4 we will not assume continuous shifting but discrete shifting, and therefore retroactively justify the results of that section.

\section{A.1. Correct clocking}

For simplicity let us define the attitude of the instrument by $\psi_{\mathrm{i}}(t)=\omega t=t$ (see Sect. 2.3). This is always possible for a satellite rotating with nominal scan rate because the zero point of the longitude coordinate on the sky can be chosen arbitrarily (see Eq. (2)). Thus the instantaneous data pixel location of a star image is $\kappa_{*}(t)=\psi_{*}-t+k(t)-\eta_{\text {tr }}$ (Eq. (6)) with $\eta_{\text {tr }}$ offset $^{7}$ from $\eta_{\text {leave }}$ by $\eta_{\text {tr }}=\eta_{\text {leave }}-\frac{1}{2}$. Without loss of generality we set $\eta_{\text {leave }}=0$; the zero point of the field-of-view angle is likewise arbitrary. The instantaneous data pixel location of the star image is therefore

$\kappa_{*}(t)=\psi_{*}+\frac{1}{2}-t+k(t)$

The data pixel location of the centroid of the star image at any time $t$ is then

$$
\begin{aligned}
\kappa_{\mathrm{c}}(t) & =\frac{1}{t-t_{\text {enter }}} \int_{t_{\text {enter }}}^{t} \kappa_{*}(\tau) \mathrm{d} \tau \\
& =\psi_{*}+\frac{1}{2}-\left.\frac{1}{2\left(t-t_{\text {enter }}\right)} \tau^{2}\right|_{t_{\text {enter }}} ^{t}+\frac{1}{t-t_{\text {enter }}} \int_{t_{\text {enter }}}^{t} k(\tau) \mathrm{d} \tau
\end{aligned}
$$

According to Fig. A.1 the integral of the step function easily can be expressed by the difference of the surface area below the step function in the interval $\left[k\left(t_{\text {enter }}\right), k\left(t_{\text {leave }}\right)+1\right]$ and the two shaded strips shown in the right-hand panel of Fig. A.1.

\footnotetext{
${ }^{7} \eta_{\text {tr }}$ and $\eta_{\text {leave }}$ differ by (nearly) half the size of one pixel because the centroids of the optical and the charge images differ by exactly the same amount, respectively. The reason for this is that the centroid of the charge image is the average of the data pixel location of the instantaneous star image over the exposure time (see also Sect. 3.3) This centroid will lie half a pixel behind the centroid of the optical image at the end of the exposure time for all stars that enter the CCD at exactly a TDI clock stroke, and very near to half a pixel behind that image for all stars entering the CCD at some arbitrary time for sufficiently large $N_{\mathrm{px}}$.
}

Therefore we find

$$
\begin{aligned}
\kappa_{\mathrm{c}}(t)= & \psi_{*}+\frac{1}{2}-\frac{t^{2}-t_{\text {enter }}^{2}}{2\left(t-t_{\text {enter }}\right)}+\frac{1}{t-t_{\text {enter }}}\left[\left(\sum_{i=k\left(t_{\text {enter }}\right)}^{k(t)} i\right)\right. \\
& \left.-\left(\left(t_{\text {enter }}-k\left(t_{\text {enter }}\right)\right) k\left(t_{\text {enter }}\right)+(k(t)+1-t) k(t)\right)\right] \\
= & \psi_{*}+\frac{1}{2}-\frac{t^{2}-t_{\text {enter }}^{2}}{2\left(t-t_{\text {enter }}\right)}+\frac{1}{t-t_{\text {enter }}}\left(\sum_{i=1}^{k(t)} i-\sum_{i=1}^{k\left(t_{\text {enter }}\right)-1} i\right) \\
& -\frac{\left(t_{\text {enter }}-k\left(t_{\text {enter }}\right)\right) k\left(t_{\text {enter }}\right)+(k(t)+1-t) k(t)}{t-t_{\text {enter }}} .
\end{aligned}
$$

Since the sum of the first $n$ natural numbers is $\frac{1}{2}\left(n^{2}+n\right)$ we can rewrite this as

$$
\begin{aligned}
\kappa_{\mathrm{c}}(t)= & \psi_{*}+\frac{1}{2}-\frac{t^{2}-t_{\text {enter }}^{2}}{2\left(t-t_{\text {enter }}\right)}+\frac{1}{t-t_{\text {enter }}} \\
& \cdot\left[\frac{1}{2}\left(k^{2}(t)+k(t)\right)-\frac{1}{2}\left(\left(k\left(t_{\text {enter }}\right)-1\right)^{2}+k\left(t_{\text {enter }}\right)-1\right)\right] \\
& -\frac{\left(t_{\text {enter }}-k\left(t_{\text {enter }}\right)\right) k\left(t_{\text {enter }}\right)+(k(t)+1-t) k(t)}{t-t_{\text {enter }}} \\
= & \psi_{*}+\frac{1}{2}-\frac{1}{2}\left(t+t_{\text {enter }}\right) \\
& +\frac{k^{2}(t)+k(t)-k^{2}\left(t_{\text {enter }}\right)+k\left(t_{\text {enter }}\right)}{2\left(t-t_{\text {enter }}\right)} \\
& -\frac{t_{\text {enter }} k\left(t_{\text {enter }}\right)-k^{2}\left(t_{\text {enter }}\right)+k^{2}(t)+k(t)-t k(t)}{t-t_{\text {enter }}} \\
= & \psi_{*}+\frac{1}{2}-\frac{1}{2}\left(t+t_{\text {enter }}\right) \\
& -\frac{k^{2}(t)+k(t)-k^{2}\left(t_{\text {enter }}\right)-k\left(t_{\text {enter }}\right)-2 t k(t)+2 t_{\text {ent. }} k\left(t_{\text {ent. }}\right)}{2\left(t-t_{\text {enter }}\right)} \\
= & \psi_{*}+\frac{1}{2}-\frac{1}{2}\left(t+t_{\text {enter }}\right)-\frac{k(t)+1-2 t}{2\left(t-t_{\text {enter }}\right)} k(t) \\
& +\frac{k\left(t_{\text {enter }}\right)+1-2 t_{\text {enter }}}{2\left(t-t_{\text {enter }}\right)} k\left(t_{\text {enter }}\right) .
\end{aligned}
$$

Fig. A. 2 shows a schematic illustration of $\kappa_{\mathrm{c}}(t)-\psi_{*}$.

In particular we are interested in the data pixel location $\kappa_{\mathrm{c}}$ of the star image at time $t_{\text {leave. }}$. Let us define a $\Delta_{t}$ such that $t_{\text {enter }}=$ $k\left(t_{\text {enter }}\right)+\Delta_{t}$. For any time $\tilde{t}=k(\tilde{t})+\Delta_{t}$ we find from Eq. (A.1)

$$
\begin{aligned}
\kappa_{\mathrm{c}}(\tilde{t})= & \psi_{*}+\frac{1}{2}-\frac{1}{2}\left(k(\tilde{t})+k\left(t_{\text {enter }}\right)+2 \Delta_{t}\right) \\
& -\frac{k(\tilde{t})+1-2 k(\tilde{t})-2 \Delta_{t}}{2\left(k(\tilde{t})-k\left(t_{\text {enter }}\right)\right)} k(\tilde{t}) \\
& +\frac{k\left(t_{\text {enter }}\right)+1-2 k\left(t_{\text {enter }}\right)-2 \Delta_{t}}{2\left(k(\tilde{t})-k\left(t_{\text {enter }}\right)\right)} k\left(t_{\text {enter }}\right) \\
= & \psi_{*}+\frac{1}{2}-\frac{1}{2}\left(k(\tilde{t})+k\left(t_{\text {enter }}\right)\right)-\Delta_{t} \\
& -\frac{1-k(\tilde{t})}{2\left(k(\tilde{t})-k\left(t_{\text {enter }}\right)\right)} k(\tilde{t})+\frac{1-k\left(t_{\text {enter }}\right)}{2\left(k(\tilde{t})-k\left(t_{\text {enter }}\right)\right)} k\left(t_{\text {enter }}\right)+\Delta_{t} \\
= & \psi_{*}+\frac{1}{2}-\frac{1}{2}\left(k(\tilde{t})+k\left(t_{\text {enter }}\right)\right) \\
& -\frac{k(\tilde{t})-k\left(t_{\text {enter }}\right)-k^{2}(\tilde{t})+k^{2}\left(t_{\text {enter }}\right)}{2\left(k(\tilde{t})-k\left(t_{\text {enter }}\right)\right)} \\
= & \psi_{*}-\frac{1}{2}\left(k(\tilde{t})+k\left(t_{\text {enter }}\right)\right)+\frac{k^{2}(\tilde{t})-k^{2}\left(t_{\text {enter }}\right)}{2\left(k(\tilde{t})-k\left(t_{\text {enter }}\right)\right)}=\psi_{*} .
\end{aligned}
$$


U. Bastian and M. Biermann: Astrometric meaning and interpretation of high-precision TDI CCD data, Online Material p 3

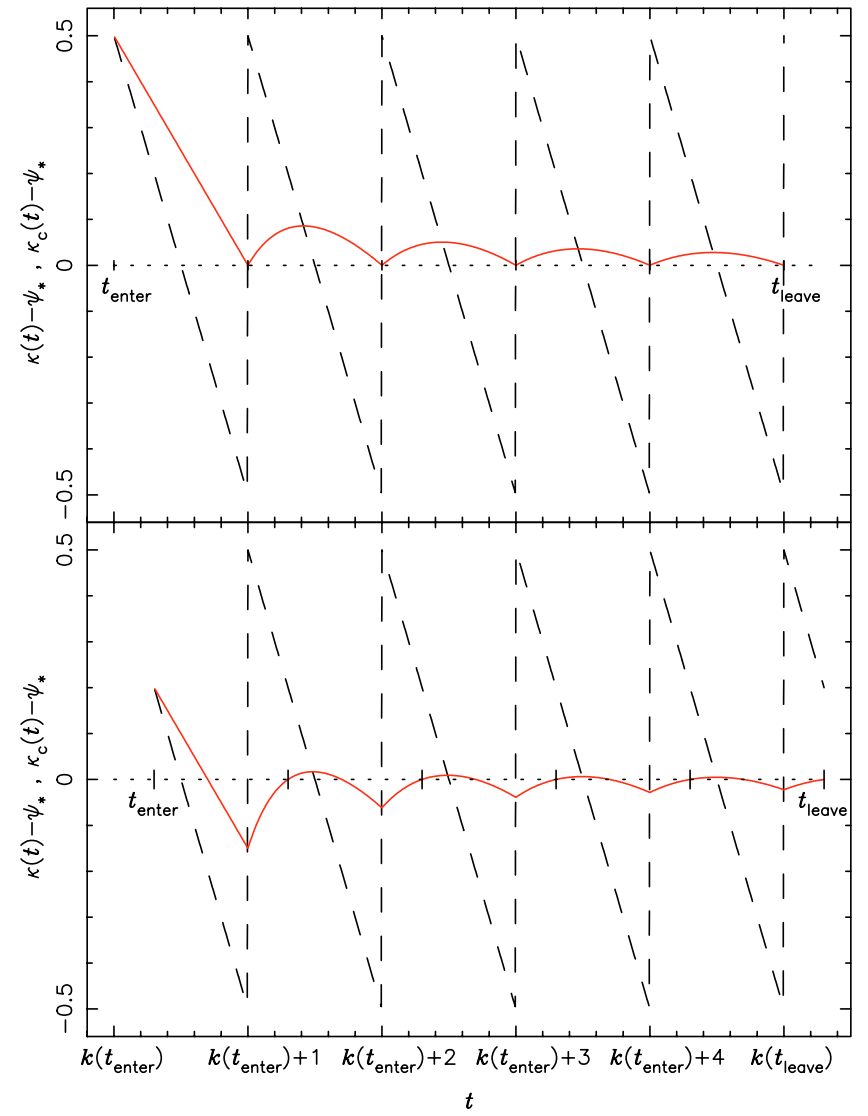

Fig. A.2. $k(t)-\psi_{*}$ (dashed) and $\kappa_{\mathrm{c}}(t)-\psi_{*}$ (solid) as a function of time $t$ for correct clocking. The dotted line indicates the result $\kappa_{\mathrm{c}}(\tilde{t})-\psi_{*}=0$ for any $\tilde{t}=t_{\text {enter }}+n, n$ a natural number. The upper plot displays the results for an integer $t_{\text {enter }}$, the bottom plot shows the results for a noninteger $t_{\text {enter }}$.

Since the exposure time is necessarily assumed to be integer (a non-integer exposure time is not possible in the case of correct clocking) we can set $\tilde{t}=t_{\text {leave }}$ and find

$\kappa_{\mathrm{c}}-\psi_{*} \equiv \kappa_{\mathrm{c}}\left(t_{\text {leave }}\right)-\psi_{*}=0$.

In the case of correct clocking and integer exposure time there are no periodic pixel effects.

\section{A.2. Incorrect clocking}

Let us now assume that the satellite is rotating slightly faster (or slower) so that the angular velocity of the satellite is $\omega=1+\Delta_{\omega}$. The instantaneous data pixel location of the star image then is

$\kappa_{*}(t)=\psi_{*}+\frac{1}{2}-\left(1+\Delta_{\omega}\right) t+k(t)$.

The computation of the data pixel location of the centroid of the star image is identical to that in Sect. A.1. Only the integration of $\psi_{\mathrm{i}}(t)$ has to be modified, but the changes are trivial. Hence we can directly write (in analogy to Eq. (A.1))

$$
\begin{aligned}
\kappa_{\mathrm{c}}(t)= & \psi_{*}+\frac{1}{2}-\frac{1+\Delta_{\omega}}{2}\left(t+t_{\text {enter }}\right)-\frac{k(t)+1-2 t}{2\left(t-t_{\text {enter }}\right)} k(t) \\
& +\frac{k\left(t_{\text {enter }}\right)+1-2 t_{\text {enter }}}{2\left(t-t_{\text {enter }}\right)} k\left(t_{\text {enter }}\right),
\end{aligned}
$$

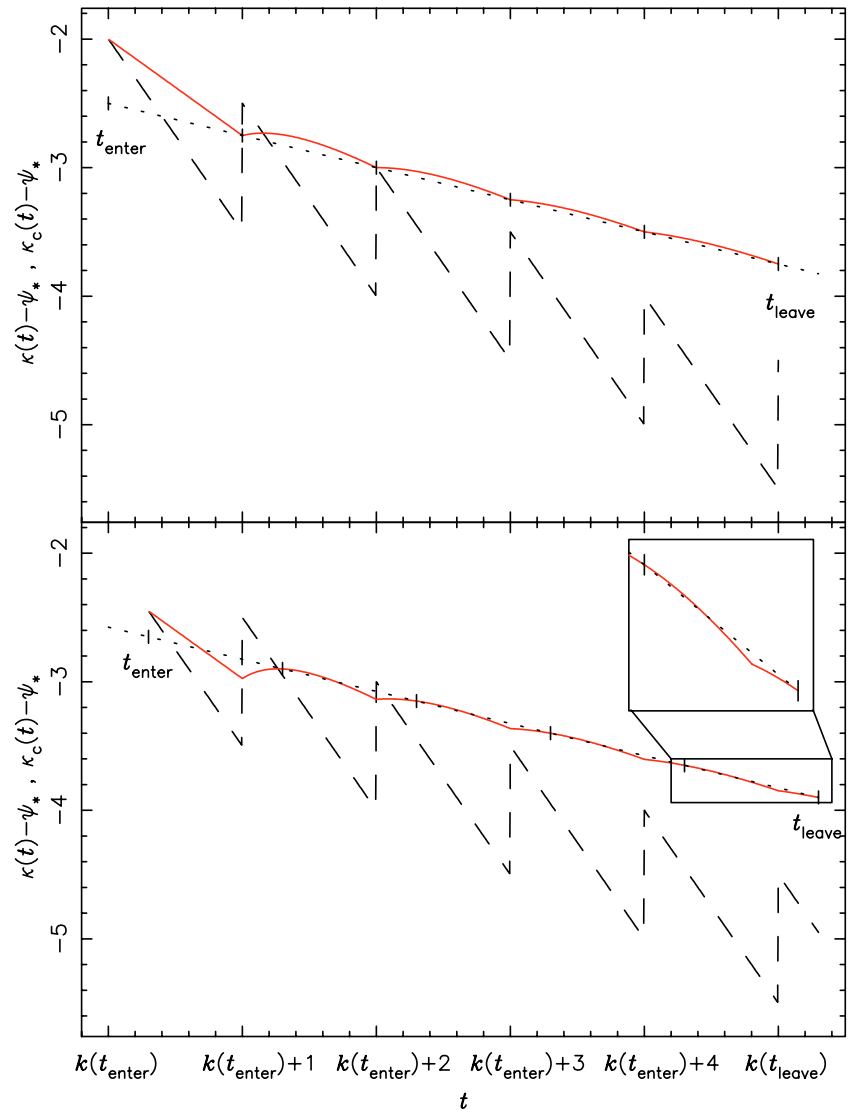

Fig. A.3. As Fig. A.2, but for incorrect clocking. Here the dotted line represents the result $\kappa_{\mathrm{c}}(\tilde{t})-\psi_{*}=\frac{\Delta_{\omega}}{2}\left(t_{\text {enter }}+t_{\text {leave }}\right)$ for any $\tilde{t}=t_{\text {enter }}+n$.

which also reduces to Eq. (A.1) for $\Delta_{\omega}=0$. Figure A.3 shows a schematic illustration of $\kappa_{\mathrm{c}}(t)-\psi_{*}$.

\section{A.2.1. Integer exposure time}

As in Sect. A.1 we can define a $\Delta_{t}$ by $\Delta_{t}=t_{\text {enter }}-k\left(t_{\text {enter }}\right)$. For any $\tilde{t}=k(\tilde{t})+\Delta_{t}$ we directly find

$\kappa_{\mathrm{c}}-\psi_{*} \equiv \kappa_{\mathrm{c}}\left(t_{\text {leave }}\right)-\psi_{*}=-\frac{\Delta_{\omega}}{2}\left(t_{\text {enter }}+t_{\text {leave }}\right)$.

The arithmetic is identical to that in Sect. A.1, only the additional term $-\frac{\Delta_{\omega}}{2}\left(t_{\text {enter }}+t_{\text {leave }}\right)$ does not vanish. So, in the case of incorrect clocking and integer exposure time there are no periodic pixel effects.

\section{A.2.2. Non-integer exposure time}

Let us - first of all - search for those combinations of $t_{\text {enter }}$ and $t_{\text {leave }}$ for which even in the case of non-integer exposure time there will be no periodic pixel effect. To find those pairs $\left(t_{\text {enter }}, t_{\text {leave }}\right)$ we have to solve the equation

$\kappa_{\mathrm{c}}\left(t_{\text {leave }}\right)-\psi_{*}+\frac{\Delta_{\omega}}{2}\left(t_{\text {leave }}+t_{\text {enter }}\right) \stackrel{!}{=} 0$.

To keep the arithmetic manageable let us define some variables. For some arbitrary times $t_{1}$ and $t_{2}$ we define the differences $\Delta_{1}$ and $\Delta_{2}$ as $\Delta_{1}=t_{1}-k\left(t_{1}\right)$ and $\Delta_{2}=t_{2}-k\left(t_{2}\right)$. Additionally we 


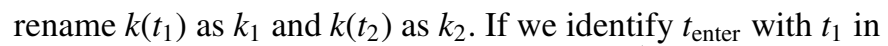
Eq. (A.3) and want to compute $\kappa_{\mathrm{c}}(t)-\psi_{*}+\frac{\Delta_{\omega}}{2}\left(k(t)+k\left(t_{1}\right)\right)$ at some time $t_{2}$, for which this expression should vanish, we find from Eq. (A.2)

$$
\begin{aligned}
0 \stackrel{!}{=} & \frac{1}{2}-\frac{1}{2}\left(k_{2}+k_{1}+\Delta_{2}+\Delta_{1}\right)-\frac{k_{2}+1-2 k_{2}-2 \Delta_{2}}{2\left(k_{2}-k_{1}+\Delta_{2}-\Delta_{1}\right)} k_{2} \\
& +\frac{k_{1}+1-2 k_{1}-2 \Delta_{1}}{2\left(k_{2}-k_{1}+\Delta_{2}-\Delta_{1}\right)} k_{1} .
\end{aligned}
$$

Multiplying this expression by the nominator $2\left(k_{2}-k_{1}+\Delta_{1}-\Delta_{2}\right)$ yields

$$
\begin{aligned}
0 \stackrel{!}{=} & \left(k_{2}-k_{1}+\Delta_{2}-\Delta_{1}\right) \\
& -\left(k_{2}+k_{1}+\Delta_{1}+\Delta_{2}\right)\left(k_{2}-k_{1}+\Delta_{2}-\Delta_{1}\right) \\
& -\left(1-k_{2}-2 \Delta_{2}\right) k_{2}+\left(1-k_{1}-2 \Delta_{1}\right) k_{1} \\
= & k_{2}-k_{1}+\Delta_{2}-\Delta_{1} \\
& -\left(k_{2}^{2}-k_{1} k_{2}+k_{2} \Delta_{2}-k_{2} \Delta_{1}+k_{1} k_{2}-k_{1}^{2}+k_{1} \Delta_{2}-k_{1} \Delta_{1}\right. \\
& \left.+k_{2} \Delta_{1}-k_{1} \Delta_{1}+\Delta_{1} \Delta_{2}-\Delta_{1}^{2}+k_{2} \Delta_{2}-k_{1} \Delta_{2}+\Delta_{2}^{2}-\Delta_{1} \Delta_{2}\right) \\
& -k_{2}+k_{2}^{2}+2 k_{2} \Delta_{2}+k_{1}-k_{1}^{2}-2 k_{1} \Delta_{1} \\
= & \Delta_{2}-\Delta_{1}-\Delta_{2}^{2}+\Delta_{1}^{2} \\
= & \left(\Delta_{2}-\Delta_{1}\right)\left(1-\left(\Delta_{2}+\Delta_{1}\right)\right) .
\end{aligned}
$$

If we return to our conventional notation $\left(t_{2}=t_{\text {leave }}\right.$ and $t_{1}=$ $\left.t_{\text {enter }}\right)$ we find that there are no pixel periodic effects in the case of incorrect clocking if and only if

1. $\Delta_{\text {leave }}=\Delta_{\text {enter }}$ : case of integer exposure time or

2. $\Delta_{\text {leave }}=1-\Delta_{\text {enter }}$.

Figure A.4 plots the term $\kappa_{\mathrm{c}}-\psi_{*}+\Delta_{\omega}\left(t_{\text {leave }}+t_{\text {enter }}\right) / 2$ as a function of $\psi_{*}$ for incorrect clocking and a non-integer exposure time. It is obvious that $\Delta t_{\text {leave }}-\Delta t_{\text {enter }}$ never vanishes and that pixel periodic effects vanish whenever the sum of the decimal places of $t_{\text {enter }}$ and $t_{\text {leave }}$ equals 1. Figure A.5 shows the expression $\kappa_{\mathrm{c}}-\psi_{*}+\Delta_{\omega}\left(t_{\text {leave }}+t_{\text {enter }}\right) / 2$ as a function of $t_{\text {enter }}$ and $t_{\text {leave }}$. The two symmetries indicated by Eq. (A.4) are clearly visible, and from the bottom plot we can directly read off the location of the maxima of the pixel periodic jitter: they are located at $\left(\Delta t_{\text {enter }}=0, \Delta t_{\text {leave }}=0.5\right)$, at $\left(\Delta t_{\text {enter }}=1, \Delta t_{\text {leave }}=0.5\right)$, at $\left(\Delta t_{\text {enter }}=0.5, \Delta t_{\text {leave }}=0\right)$ and at $\left(\Delta t_{\text {enter }}=0.5, \Delta t_{\text {leave }}=1\right)$. With this knowledge we can calculate the magnitude of this pixel periodic effect. To see the dependence on the CCD size, i.e. $N_{\mathrm{px}}$, we set $k\left(t_{\mathrm{enter}}\right)=0$ (which is always possible) and replace $t_{\text {leave }}$ by $k\left(t_{\text {leave }}\right)+\Delta_{\text {leave }}=N_{\text {px }}+\Delta_{\text {leave }}$ for sufficiently small deviations from the nominal scan speed. Thus we can rewrite Eq. (A.2) in the form

$$
\begin{aligned}
& \kappa_{\mathrm{c}}\left(t_{\text {leave }}\right)-\psi_{*}+\frac{\Delta_{\omega}}{2}\left(t_{\text {leave }}+t_{\text {enter }}\right) \\
& =\frac{1}{2}-\frac{1}{2}\left(\Delta_{\text {enter }}+N_{\mathrm{px}}+\Delta_{\text {leave }}\right) \\
& -\frac{N_{\mathrm{px}}+1-2 N_{\mathrm{px}}-2 \Delta_{\text {leave }}}{2\left(N_{\mathrm{px}}+\Delta_{\text {leave }}-\Delta_{\text {enter }}\right)} N_{\mathrm{px}} .
\end{aligned}
$$

Inserting one of the maximum pixel periodic effect locations (e.g. $\Delta_{\text {enter }}=0$ and $\Delta_{\text {leave }}=0.5$ ) we find a maximum possible effect of

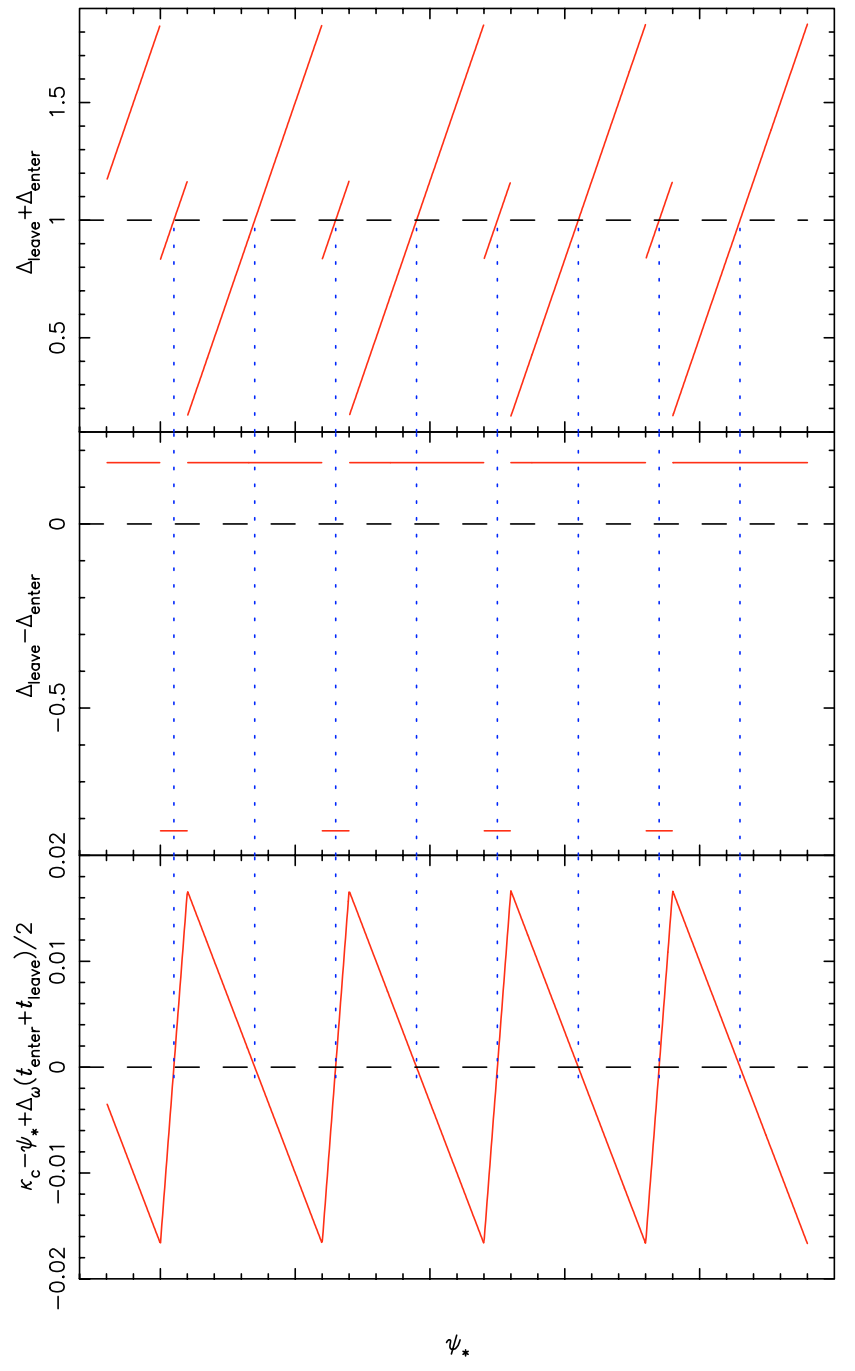

Fig. A.4. $\Delta t_{\text {leave }}+\Delta t_{\text {enter }}($ top $), \Delta t_{\text {leave }}-\Delta t_{\text {enter }}($ middle $)$ and $\kappa_{\mathrm{c}}-\psi_{*}+$ $\Delta_{\omega}\left(t_{\text {leave }}+t_{\text {enter }}\right) / 2$ (bottom) as a function of $\psi_{*}$ for incorrect clocking and a non-integer exposure time.

$$
\begin{aligned}
& \kappa_{\mathrm{c}}\left(t_{\text {leave }}\right)-\psi_{*}+\frac{\Delta_{\omega}}{2}\left(t_{\text {leave }}+t_{\text {enter }}\right) \\
& =\frac{1}{2}\left(1-N_{\mathrm{px}}-\frac{1}{2}+\frac{N_{\mathrm{px}}^{2}}{N_{\mathrm{px}}+\frac{1}{2}}\right) \\
& =\frac{1}{2}\left(1-N_{\mathrm{px}}-\frac{1}{2}+\frac{\left(\left(N_{\mathrm{px}}+\frac{1}{2}\right)-\frac{1}{2}\right)^{2}}{N_{\mathrm{px}}+\frac{1}{2}}\right) \\
& =\frac{1}{2}\left(1-N_{\mathrm{px}}-\frac{1}{2}+N_{\mathrm{px}}+\frac{1}{2}-1+\frac{\left(\frac{1}{2}\right)^{2}}{N_{\mathrm{px}}+\frac{1}{2}}\right) \approx \frac{1}{8 N_{\mathrm{px}}} .
\end{aligned}
$$

Note that $N_{\mathrm{px}}$ in this consideration is the total number of charge transfer steps along scan, not the total number of physical pixels (as already briefly noted in Footnote 1). For large $N_{\mathrm{px}}$ the effect is negligible compared to the errors that arise during the data reduction process and to any conceivable measurement noise level. In case of Gaia the maximum TDI-induced periodic jitter is $0.31 \mu$ as for the Astro CCDs and $35 \mu$ as for the Spectro CCDs (corresponding to $0.5 \mathrm{~km} \mathrm{~s}^{-1}$ in radial velocity). Both effects are negligible compared to the measurement precision from individual CCD transits. 
U. Bastian and M. Biermann: Astrometric meaning and interpretation of high-precision TDI CCD data, Online Material p 5
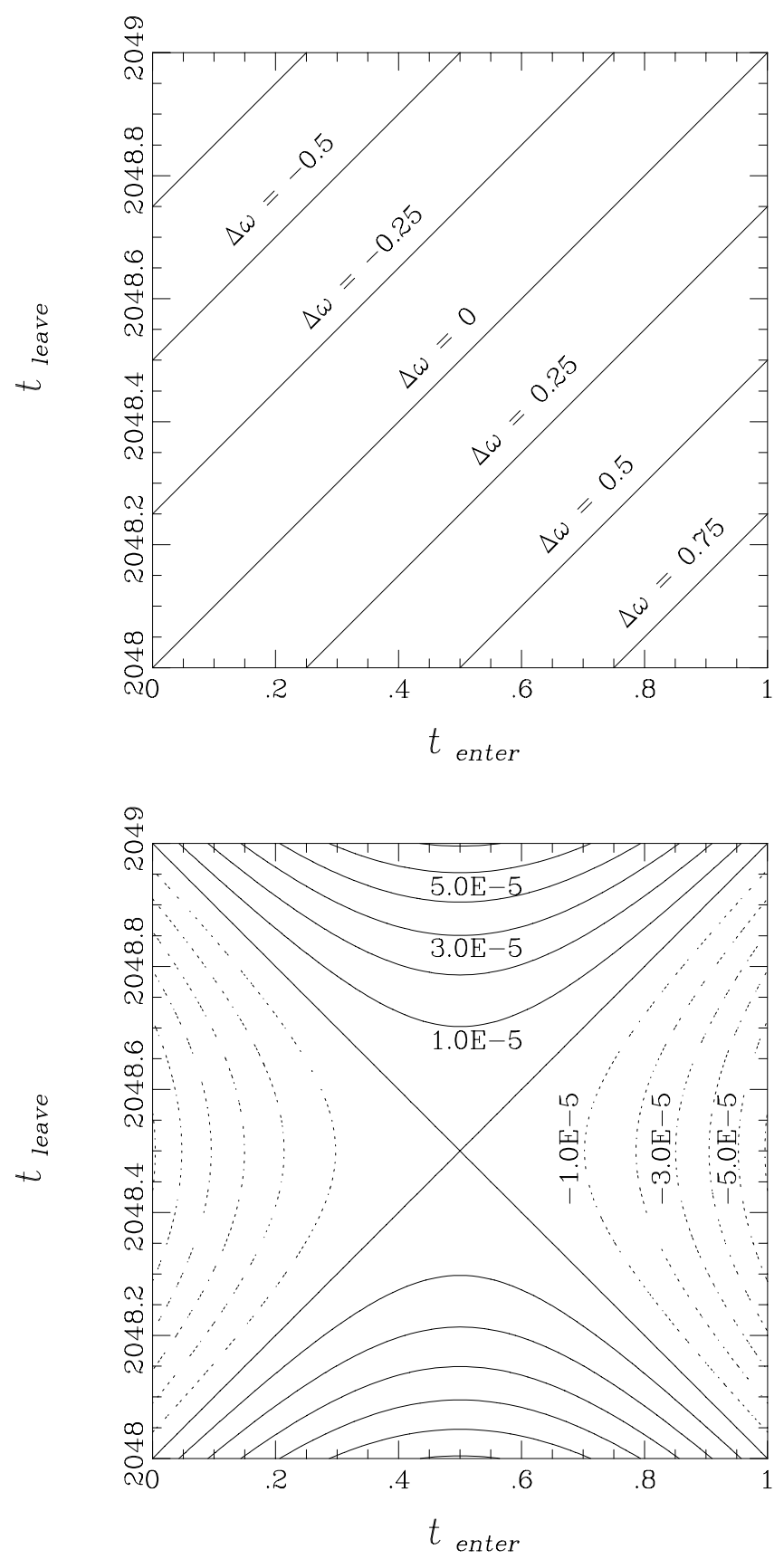

Fig. A.5. $\kappa_{\mathrm{c}}-\psi_{*}+\Delta_{\omega}\left(t_{\text {leave }}+t_{\text {enter }}\right) / 2$ (bottom) as a function of $t_{\text {enter }}$ and $t_{\text {leave }}$ for a CCD with 2048 transfer steps in scan direction. The time at which the star image enters the CCD is set to lie between 0 and 1 which is always possible. The sketch at the top is to illustrate the distribution of $\Delta \omega$ in the $t_{\text {enter }}-t_{\text {leave }}$-plane.

For optical images (point-spread functions) spanning several pixels the actual pixel-periodic jitter will be smaller than the maximum effect given by Eq. (A.5), due to averaging over different parts of the image. This is why the pixel-periodic jitter will be negligible even for Astro measurements using gates (i.e. much fewer pixels).

\section{Appendix B: Computation of Spectro attitude from Astro attitude is not possible}

In this appendix we prove that the computation of the astrometric attitude for the Spectro instrument from the astrometric attitude of the Astro instrument is not possible, even in the absence of any noise.

Let us denote the astrometric attitudes of the two instruments by $\psi_{\mathrm{c}}^{\text {Astro }}$ and $\psi_{\mathrm{c}}^{\text {Spectro }}$. From Sect. 3.4 (Eq. (12)) we know that these attitudes are the inverse functions of $\kappa_{\mathrm{c}}^{\text {Astro }}\left(\psi_{*}\right)$ and $\kappa_{\mathrm{c}}^{\text {Spectro }}\left(\psi_{*}\right)$, respectively. So, to prove that one $\kappa_{\mathrm{c}}$ cannot be computed from the other would imply that one attitude cannot be computed from the other. So, we can consider the simpler problem for $\kappa_{\mathrm{c}}^{\text {Astro }}\left(\psi_{*}\right)$ and $\kappa_{\mathrm{c}}^{\text {Spectro }}\left(\psi_{*}\right)$.

By using Eq. (10) as the definition of $\kappa_{\mathrm{c}}$, by inserting the definition of $\kappa_{*}$ from Eq. (5), and by assuming nominal TDI clocking $k(t)=t$, we find (ignoring the normalisation factor of the exposure function $e(t)$ and the constant $\kappa_{0}$ for simplicity):

$$
\begin{aligned}
\kappa_{\mathrm{c}}\left(\psi_{*}\right) & =\int_{-\infty}^{+\infty} e\left(t-\psi_{*}\right) \kappa_{*}(t) \mathrm{d} t \\
& =\int_{-\infty}^{+\infty} e\left(t-\psi_{*}\right)\left[\psi_{*}-\psi_{\mathrm{i}}(t)+k(t)\right] \mathrm{d} t \\
& =\psi_{*}-\int_{-\infty}^{+\infty} e\left(t-\psi_{*}\right) \psi_{\mathrm{i}}(t) \mathrm{d} t+\int_{-\infty}^{+\infty} e\left(t-\psi_{*}\right) t \mathrm{~d} t .
\end{aligned}
$$

Here we have two trivial terms, and one containing the actual physical attitude $\psi_{\mathrm{i}}(t)$. Ignoring the two trivial ones we can conclude that $\kappa_{\mathrm{c}}\left(\psi_{*}\right)$ is essentially determined by the convolution of the actual physical attitude with the exposure function(s), in other words, by the average of the physical attitude over the effective exposure time interval for a star at $\psi_{*}$.

In the Fourier domain the convolution transforms into a product. If we denote the Fourier transforms of the functions $\kappa, e, \psi$ by the respective upper-case letters $\mathrm{K}, \mathrm{E}, \Psi$ we can write (again ignoring normalisation factors)

$\mathrm{K}_{\mathrm{c}}^{\text {Astro }}=\mathrm{E}^{\text {Astro }} \Psi_{\mathrm{i}}$ and $\mathrm{K}_{\mathrm{c}}^{\text {Spectro }}=\mathrm{E}^{\text {Spectro }} \Psi_{\mathrm{i}}$,

from which follows

$\mathrm{K}_{\mathrm{c}}^{\text {Spectro }}=\mathrm{K}_{\mathrm{c}}^{\text {Astro }} \frac{\mathrm{E}^{\text {Spectro }}}{\mathrm{E}^{\text {Astro }}}$.

This equation shows that the transformation from Astro to Spectro can only be done if either $\mathrm{E}^{\text {Astro }}$ has no zeroes, or if all zeroes of $\mathrm{E}^{\text {Astro }}$ are also zeroes of $\mathrm{E}^{\text {Spectro }}$. For box-shaped $\mathrm{E}^{\text {Astro }}$ and $\mathrm{E}^{\text {Spectro }}$ the Fourier transforms are scaled $\sin (\omega) / \omega$ functions, where $\omega$ denotes the frequency argument in the Fourier domain. Each of them has an infinite series of zeroes (at $\omega=n \pi / t_{\text {exp }}$ for all integer numbers $n$ except zero). These zeroes obviously are at different $\omega$, because the two $t_{\exp }$ are different (and not simple integer multiples of each other).

Thus the last equation above cannot be solved, even in the absence of noise. As an aside it should be mentioned that assuming exposure functions which are not box-shaped (but take the actual image structure into account) would only produce more zeroes in the Fourier transforms.

These considerations also hold for the computation of the astrometric attitude for Astro observations using gates (i.e. for the bright stars) from the high-precision Astro attitude. 